\title{
Maximizing Information on the Environment by Dynamically Controlled Qubit Probes
}

\author{
Analia Zwick, Gonzalo A. Álvarez, and Gershon Kurizki \\ Weizmann Institute of Science, Rehovot 76100, Israel \\ (Received 29 July 2015; revised manuscript received 16 October 2015; published 25 January 2016) \\ We explore the ability of a qubit probe to characterize unknown parameters of its environment. By \\ resorting to the quantum estimation theory, we analytically find the ultimate bound on the precision of \\ estimating key parameters of a broad class of ubiquitous environmental noises ("baths") which the qubit \\ may probe. These include the probe-bath coupling strength, the correlation time of generic types of bath \\ spectra, and the power laws governing these spectra, as well as their dephasing times $T_{2}$. Our central result \\ is that by optimizing the dynamical control on the probe under realistic constraints one may attain the \\ maximal accuracy bound on the estimation of these parameters by the least number of measurements \\ possible. Applications of this protocol that combines dynamical control and estimation theory tools to \\ quantum sensing are illustrated for a nitrogen-vacancy center in diamond used as a probe.
}

DOI: 10.1103/PhysRevApplied.5.014007

\section{INTRODUCTION}

Controlled spin- $\frac{1}{2}$ particles (qubits) are sensitive probes of the structure and properties of highly complex molecular, atomic, or solid-state quantum systems. Quantum technologies requiring such high sensitivity at the nanoscale are based on qubit probes serving as sensors [1-8] or monitors of biological or chemical processes [9-11]. Here we focus on the extraction of information characterizing the environment of a qubit probe, by monitoring the decoherence process that the qubit undergoes [12]. Dynamical control, originally conceived as a tool for reducing decoherence effects [13-19], is also shown to be a valuable source of information on environmental noises [10,11,20-25]. This information is revealed by the dependence of the decoherence rate of the qubit probe on a control-field parameter, owing to the fact that, under weak coupling of the qubit and the environment ("bath"), this rate is universally expressed by the overlap of the environmental noise spectrum and a spectral filter function that is solely determined by the dynamical control $[15-17,26]$. The filter function can then be designed by varying the control field to scan the noise spectrum. The information obtained from this procedure, dubbed "noise spectroscopy" [22,23], is not only essential for designing the most effective (optimal) dynamical protection from decoherence caused by a given environment [26-33] of quantum-information processing [34-37], quantum-state transfer [38,39], and quantum-state storage [40,41]. It may also become a means of understanding physical or chemical processes $[9-11,24]$ by analyzing their noise fluctuations in magnetic resonance spectroscopy and imaging with nanoscale resolution [6-8,11,42-44].

In order to further advance this promising noise spectroscopy and broaden its applicability, it is imperative to find the best general strategy for extracting the environmental ("bath-induced") noise-spectrum information from the qubit-probe decoherence. The strategy we adopt aims at minimizing the error in estimating key parameters of the noise (bath) spectrum by measurements performed on the qubit probe (Sec. II). The minimal error is determined by the maximal quantum Fisher information (QFI) [45-51] gathered by measurements in the optimal basis (Sec. III). For parameters that characterize generic types of environmental noise, we here find (Sec. III A) the ultimate error bounds for unbiased estimators when the qubit probe, under arbitrary control, undergoes pure dephasing in the probe-bath weak-coupling regime. Motivated by practical experimental considerations and constraints, these ultimate bounds attain the best estimation precision by the least number of measurements possible. We achieve these goals by replacing the free-evolution (-induction) decay (FID) of the qubit state prior to each measurement by dynamically controlled evolution designed to ensure the convergence to the ultimate error bound for the particular bath-spectrum parameter to be estimated (Sec. III B). For each such parameter, an appropriate filter function must be generated by dynamical control (Sec. IV A) $[17,26]$. We here focus on determining the general conditions that have to be satisfied for designing the filter function to attain the ultimate bounds.

The first step in the proposed strategy is the estimation of the coupling strength $g$ of the bath to the probe that can be interpreted as the noise variance. The proposed appropriate filter function is generated by projections onto an eigenstate of the $\sigma_{x}$ probe operator at a rate that conforms to the quantum Zeno regime [15,24,52] (Sec. IV B). This procedure, which does not require prior knowledge of the bath spectral line shape, has been experimentally exploited to determine the coupling strengths of complex spin networks but without maximizing or optimizing the information obtained [24]. We note that our approach differs from 
approaches for phase estimation [53] whereby the phase is deduced from a coherent oscillation of the probe induced by the coupling strength $g$. In our scheme, the phase induced by $g$ is a stochastic variable associated with an incoherent evolution of the probe.

Once the coupling strength $g$ is optimally estimated, the bath spectra have to be characterized by their normalized line shapes. These spectra crucially depend on the bath correlation time $\tau_{c}$ often unknown to us: It is typically the inverse of $\omega_{c}$, which is the width or the cutoff of the bath spectrum. We show that a convergence to the lowest error bound on $\tau_{c}$ is achievable through filter functions generated by common types of coherent dynamical-control sequences (Sec. IV C). By contrast, FID of the qubit coherence $\left\langle\sigma_{x}(t)\right\rangle$ may preclude such a convergence.

We further show for a family of generic baths that optimized convergence requires a filter function that only samples (overlaps) a power-law region of the bath spectrum (Sec. III B). Such spectral features characterize omnipresent baths: sub-Ohmic, Ohmic, and super-Ohmic baths whose spectra obey a power law at low frequencies, as well as noise spectra of generalized Ornstein-Uhlenbeck processes characterized by a power-law tail at high frequencies. These types of bath spectra are ubiquitous in solid-state, liquid, or gas phases [11,23,54-57], where they represent collisional or diffusion processes $[58,59]$. Other environmental parameters, such as spectral powerlaw exponents, the $T_{2}$ decoherence time, and diffusion coefficients, are shown to obey bounds analogous to those of $g$ and $\tau_{c}$ (Sec. IV D). The ultimate bounds obtained here are instrumental for improving the estimation of parameters that characterize dephasing, which have been previously inferred under free evolution [48,50].

Finally, we demonstrate the practical feasibility of experiments that may attain the ultimate precision bounds by resorting to a real-time adaptive-estimation protocol based on a Bayesian estimator and an online experimental learning design [60-63] (Sec. V). We resort to this protocol to illustrate the ability to achieve the predicted analytical bounds in an efficient way under experimentally relevant conditions, e.g., for nitrogen-vacancy center (NVC) probes in diamond [1-8,44,54]. Thus, the present analytical theory, supported by adaptive-estimation simulations, suggests that the proposed synthesis of optimally controlled noise spectroscopy and estimation theory can become a powerful, broadly applicable, diagnostic tool (Sec. VI).

In summary, we demonstrate that the ultimate accuracy bound on environmental (noises) characteristics can be achieved by optimizing the dynamical control on the probe under realistic constraints and optimal timing of the control pulses. Moreover, to simulate experiments that maximize the information gain, we put forward a real-time adaptiveestimation protocol that attains the predicted bounds in an efficient way. These results open the door to the development of an important diagnostic tool of environmental processes by qubit probes. Such diagnostics are expected to become a key element in diverse technological applications: nuclear magnetic resonance (NMR), magnetic resonance imaging (MRI), optical spectroscopy, and imaging of chemical and biomedical processes [6,7,11,42-44,6466] to mention a few.

\section{DEPENDENCE OF THE QUBIT-PROBE DEPHASING ON AN UNKNOWN ENVIRONMENTAL PARAMETER}

We consider a qubit probe that experiences proper dephasing by the environment (bath) under the action of the system-bath interaction Hamiltonian

$$
H_{\mathrm{SB}}=g \sigma_{z} B
$$

where $g$ is the probe-bath interaction strength, $\sigma_{z}$ is the appropriate Pauli operator for the probe, and $B$ is the bath operator [Fig. 1(a)]. To obtain maximal information on the environment, a convenient initial probe state is the symmetric superposition of the qubit-up and -down states in the $\sigma_{z}$ basis,

$$
\frac{1}{\sqrt{2}}(|\uparrow\rangle+|\downarrow\rangle)=|+\rangle,
$$

and the optimal observable for the probe-state measurement is $\sigma_{x}$ [48] (Appendix B). Our goal is to determine the dynamical-control efficacy for estimating, one by one, the unknown parameters such as $g$ and $\tau_{c}$ that characterize the coupling bath spectrum.

We denote the particular bath parameter by $x_{B}$ and, accordingly, the bath coupling spectrum by $G\left(x_{B}, \omega\right)$. Under proper dephasing,

$$
\left\langle\sigma_{x}\left(x_{B}, t\right)\right\rangle=\operatorname{Tr}\left[\rho_{S}\left(x_{B}, t\right) \sigma_{x}\right]=e^{-\mathcal{J}\left(x_{B}, t\right)},
$$

where $\mathcal{J}\left(x_{B}, t\right)$ is the attenuation factor due to dephasing. In the probe-bath weak-coupling regime known as the Born approximation for the bath, wherein the qubit probe negligibly influences the environment [67], it obeys the universal formula (Appendix A) [15-17,26]

$$
\mathcal{J}\left(x_{B}, t\right)=\int_{-\infty}^{\infty} d \omega F_{t}(\omega) G\left(x_{B}, \omega\right),
$$

where $F_{t}(\omega)$ is a filter function which explicitly depends upon the dynamical control of the qubit probe during time $t$.

This universal formula is exact for Gaussian noise. Yet it applies to any noise under the weak-coupling assumption in Eq. (4). In our bath-optimized control theory [15-17,26,27], the filter function is obtained to ensure optimal control for any given bath and task at hand. This theory does not impose any requirement on the temporal shape of dynamical control that defines the filter function: 

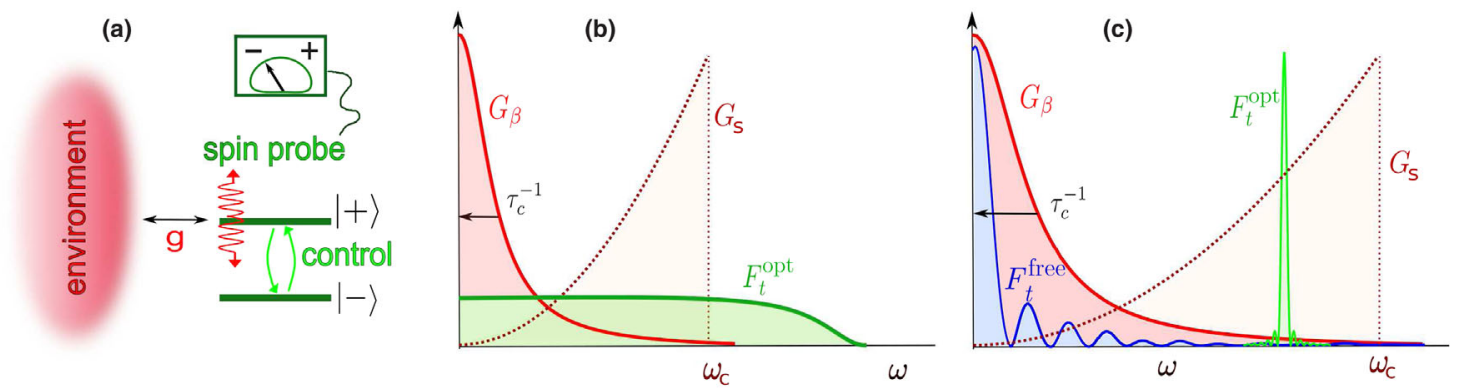

FIG. 1. Scheme of noise-spectra parameter estimation. (a) Scheme of the probing process and its dynamical control: A dynamically controlled qubit probe undergoes dephasing by the environment (bath). The coupling strength $g$ of the probe qubit and the environment affects the energy splitting between the symmetric and antisymmetric qubit states $| \pm\rangle=(1 / \sqrt{2})(|\uparrow\rangle \pm|\downarrow\rangle)$. (b) An optimal qubit-probe filter function $F_{t}^{\text {opt }}(\omega)$ generated by frequent projections is spectrally flat to the extent that it conforms to be quantum Zeno regime and allows the optimal determination of the coupling strength $g$ regardless of the shape of $G(\omega)=g^{2} S(\omega)$. (c) A filter function of an optimally controlled qubit probe, $F_{t}^{\mathrm{opt}}(\omega)$, for obtaining the ultimate bound on the correlation time $\left(\tau_{c}\right)$ estimation. Such a filter must overlap only with the power-law tail of the Ornstein-Uhlenbeck noise spectrum $S_{\beta}\left(\tau_{c}, \omega\right)$, or with $\omega>0$ but not with $\omega_{c}$ for the (super-) Ohmic spectrum $S_{s}\left(\tau_{c}, \omega\right)$. The FID filter function $F_{t}^{\text {free }}(\omega)$ does not fulfill the requirements for achieving the bound, since it is centered at $\omega=0$.

The control may be continuous or pulsed, coherent or projective.

The information about the unknown bath parameter $x_{B}$ is encoded in the protocol defined by Eqs. (1)-(4) (Fig. 1) by the probabilities $p$ of finding the qubit in the $|+\rangle$ (symmetric) or $|-\rangle$ (antisymmetric) state when measuring $\sigma_{x}$. These probabilities obey

$$
p\left( \pm \mid x_{B}, t\right)=\frac{1}{2}\left(1 \pm e^{-\mathcal{J}\left(x_{B}, t\right)}\right) .
$$

\section{OPTIMAL ESTIMATION UNDER DYNAMICAL CONTROL}

The minimum achievable relative error of the unbiased estimation of a single unknown parameter $x_{B}$ is determined by the quantum Cramer-Rao bound to be

$$
\varepsilon\left(x_{B}, t\right)=\frac{\delta x_{B}}{x_{B}} \geq \frac{1}{x_{B} \sqrt{N_{m} \mathcal{F}_{\mathcal{Q}}\left(x_{B}, t\right)}},
$$

where $N_{m}$ is the number of measurements and $\mathcal{F}_{\mathcal{Q}}\left(x_{B}, t\right)$ is the QFI that quantifies the maximum amount of information on $x_{B}$ that can be extracted from a given state [46]. Therefore, we set out to maximize the information

$$
\mathcal{F}_{\mathcal{Q}}\left(x_{B}, t_{\text {opt }}\right)=\max _{t} \mathcal{F}_{\mathcal{Q}}\left(x_{B}, t\right)
$$

by choosing the optimal time $t_{\mathrm{opt}}$ to perform the measurement and an appropriate dynamical-control scheme (prior to the measurement) to obtain the minimal attainable (relative) error in the estimation of $x_{B}$ for a given bath spectrum. For a qubit probe obeying Eq. (5), the QFI is gathered by measurements in the optimal basis (Appendix B)

$$
\mathcal{F}_{\mathcal{Q}}\left(x_{B}, t\right)=\frac{e^{-2 \mathcal{J}\left(x_{B}, t\right)}}{1-e^{-2 \mathcal{J}\left(x_{B}, t\right)}}\left(\frac{\partial \mathcal{J}\left(x_{B}, t\right)}{\partial x_{B}}\right)^{2} .
$$

From this expression, the QFI maximum is obtained by finding the optimal tradeoff between the signal-amplitude contrast (the magnitude of $e^{-2 \mathcal{J}} /\left(1-e^{-2 \mathcal{J}}\right)$ ) and the sensitivity of the signal attenuation to the parameter $x_{B}$ (the derivative $\partial \mathcal{J} / \partial x_{B}$ ). Obviously, neither should be too small if Eq. (8) is to be maximized. Their optimal tradeoff determines $t_{\text {opt }}$ for a given control scheme [Fig. 2(a)]. To evaluate the efficacy of different dynamical controls in attaining the highest accuracy in $x_{B}$ and thereby find the most suitable control schemes, we then use the error (6) at $t_{\text {opt }}$, i.e., $\varepsilon\left(x_{B}, t_{\text {opt }}\right)$, as the figure of merit.

We focus here on the ultimate error bounds for the estimation precision per measurement for the qubit probe that undergoes pure dephasing within the weak-coupling regime. These bounds are of practical importance in typical experimental situations where the initialization and readout times, normally determined by $T_{1} \gg T_{2}$, constrain the time interval between measurements.

Alternatively, in instances where $T_{1}$ is not the dominant constraint, one may be interested in the ultimate precision bound attainable during a given interrogation time that extends over many measurements, $T=N_{m} t$. In such cases, the Fisher information per unit time has to be maximized:

$$
\mathcal{F}_{\mathcal{Q}}\left(x_{B}, t_{\mathrm{opt}}\right) / t_{\mathrm{opt}}=\max _{t}\left[\mathcal{F}_{\mathcal{Q}}\left(x_{B}, t\right) / t\right] .
$$

\section{A. Ultimate estimation bounds}

As discussed below, a broad class of practically relevant environmental noise processes cause the attenuation factor $\mathcal{J}\left(x_{B}, t\right)$ to have a power-law dependence in $x_{B}$ with an exponent $\alpha$, where the derivative of $\mathcal{J}\left(x_{B}, t\right)$ is tightly bounded by its value in the power-law region, as 

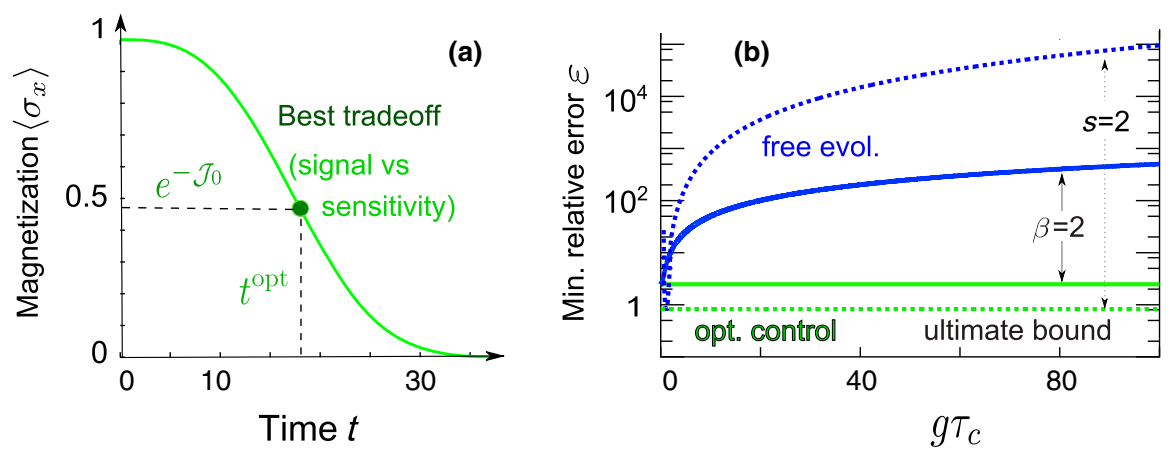

FIG. 2. Optimization for achieving the ultimate precision bound. (a) Magnetization $\left\langle\sigma_{x}\right\rangle$ as a function of the measurement time $t$ of a qubit probe experiencing dephasing due to the Ornstein-Uhlenbeck process, with Lorentzian spectrum $G_{\beta=2}\left(\tau_{c}=10, \omega\right)$ with $g=1$ under a Carr-Purcell-Meiboom-Gill (CPMG) control sequence with $N=8$ pulses using the exact analytical expression derived from Eq. (4) [11]. For the particular control, the optimal time $t^{\text {opt }}$ that determines the best tradeoff between the signal contrast (almost halfway between 0 and 1) and the highest sensitivity of the decay rate to $\tau_{c}$ provides the most accurate estimation of $\tau_{c}$. (b) Minimal relative error $\varepsilon\left(\tau_{c}, t^{\mathrm{opt}}\right)$ per measurement $\left(N_{m}=1\right)$ of the estimation of $\tau_{c}$ as a function of $g \tau_{c}$ for a Lorentzian spectrum $G_{\beta=2}\left(\tau_{c}=10, \omega\right)($ dashed lines) and a super-Ohmic spectrum $G_{s=2}\left(\tau_{c}=10, \omega\right)$ (solid lines) calculated from the integral of Eq. (4), both with $g=1$. The minimal relative error under optimal control [CPMG $(\mathrm{CW})$ with $N$ pulses (cycles) satisfying the conditions given in the main text, green lines] achieves the ultimate bounds $\left[\varepsilon_{0} / 3 \sqrt{N_{m}}\right.$ (dashed) and $\varepsilon_{0} / \sqrt{N_{m}}$ (solid) green lines for $s=2$ and $\beta=2$, respectively]. It can improve the best precision obtainable under free evolution (blue lines) by several orders of magnitude.

$$
\left|\frac{\partial \mathcal{J}\left(x_{B}, t\right)}{\partial x_{B}}\right| \leq \frac{\alpha \mathcal{J}\left(x_{B}, t\right)}{x_{B}}
$$

The equality strictly holds when $\mathcal{J}\left(x_{B}, t\right)$ is a homogeneous function of degree $\alpha$ for the parameter $x_{B}$.

The bound (10) leads to a tight lower bound on the relative error in (6) (Appendix C):

$$
\varepsilon\left(x_{B}, t\right) \geq \frac{\sqrt{1-e^{-2 \mathcal{J}_{0}}}}{\alpha \sqrt{N_{m}} \mathcal{J}_{0} e^{-\mathcal{J}_{0}}}=\frac{\varepsilon_{0}}{\alpha \sqrt{N_{m}}} .
$$

Here the value of $\mathcal{J}=\mathcal{J}_{0}$ that minimizes this bound, $\mathcal{J}_{0}=1+\frac{1}{2} W\left(-2 e^{-2}\right) \approx 0.8, W(z)$ being the Lambert function, yields the equality in (11), with $\varepsilon_{0} \approx 2.48$. We have thus obtained a universal, ultimate error bound for the unbiased estimation of $x_{B}$ in a broad class of noise spectra probed by a qubit probe undergoing dephasing within the probe-bath weak-coupling regime. This bound is universal and ultimate in the sense that it holds for arbitrary dynamical control or FID of the probe coherence and is independent of the specific environment under the assumptions discussed above.

\section{B. Key parameter estimation}

This general bound applies to the estimation of any parameters that satisfy $\mathcal{J}\left(x_{B}, t\right) \propto x_{B}^{ \pm \alpha}$. We here discuss in detail some examples of estimating key parameters that belong to this class in typical scenarios of practical importance.

(i) The effective coupling strength $g$ of the qubit probe with the dephasing bath is required for defining

$$
G\left(x_{B}=g, \omega\right)=g^{2} S(\omega),
$$

$S(\omega)$ being the normalized spectral density of the bath autocorrelation function, so that $G(g, \omega)$ and $\mathcal{J}(g, t)$ are homogeneous functions of degree $\alpha=2$ in $g$.

(a) The correlation time $\tau_{c}$ of noise fluctuations is required for describing generalized OrnsteinUhlenbeck processes, with normalized spectral densities

$$
S_{\beta}\left(x_{B}=\tau_{c}, \omega\right)=\mathcal{A}_{\beta} \frac{\tau_{c}}{1+\omega^{\beta} \tau_{c}^{\beta}},
$$

where $\beta \geq 2$ is an even integer and $\mathcal{A}_{\beta}=$ $(\beta / 2 \pi) \sin (\pi / \beta)$ is the normalization factor.

(b) The correlation time $\tau_{c}=\omega_{c}^{-1}$, that is the inverse of the cutoff frequency $\omega_{c}$, in generalized Ohmic spectra

$$
\begin{aligned}
& S_{s}\left(x_{B}=\tau_{c}, \omega\right) \\
& \quad=(s+1) \omega_{c}^{-(s+1)} \omega^{s} \Theta(\omega) \Theta\left(\omega-\omega_{c}\right),
\end{aligned}
$$

where $\Theta(\omega)$ is the step function, $s=1$ stands for an Ohmic spectrum, $0<s<1$ for a sub-Ohmic spectrum, and $s>1$ for its super-Ohmic counterpart.

Both spectral densities (13) and (14) satisfy $\left|\partial S / \partial \tau_{c}\right| \leq\left(\alpha S / \tau_{c}\right)$ and, consequently, the bound (10). Furthermore, both spectra attain the ultimate bound (11) at frequency ranges where they have power-law dependence (Appendix C): spectral density (13) at high frequencies, where it becomes a homogeneous function of degree $\alpha=\beta-1$, and 
spectral density (14) at low frequencies, when we restrict ourselves to $\omega<\omega_{c}$ to avoid the cutoff effects, thus rendering the spectral density a homogeneous function of degree $\alpha=s+1$.

(iii) The dephasing time $T_{2}$ in the attenuation exponent which is a homogeneous function of degree $\alpha$, $\mathcal{J}\left(x_{B}=T_{2}, t\right)=\left(t / T_{2}\right)^{\alpha}$, may be estimated down to the ultimate bound.

\section{DYNAMICAL-CONTROL STRATEGIES FOR ACHIEVING THE ULTIMATE BOUND}

\section{A. Achieving the ultimate bound by dynamical control}

It follows from the discussion above that the ultimate error bound, Eq. (11), in the estimation of $x_{B}=g$ or $\tau_{c}$, can be attained only provided that first the dynamical control on the probe generates a filter $F_{t}(\omega)$ that extends only over the frequency band where the noise spectrum behaves as a power law in $x_{B}^{ \pm \alpha}$ and the equality in Eq. (10) is fulfilled. Then, upon adjusting the total control time such that $\mathcal{J}\left(x_{B}, t^{\text {opt }}\right)=\mathcal{J}_{0}$, the equalities in Eq. (11) are also fulfilled.

We note that these are general conditions for attaining the ultimate error bound per measurement, but they do not explicitly invoke the optimization of the filter function. Under specific constraints that may be imposed in a given experimental setup, further optimization may be required to approach as best we can the ultimate error bounds.

\section{B. Estimating the probe-bath interaction strength $g$}

If $S(\omega)$ is unknown apart from a crude estimate of its overall width, dynamical control is needed for estimating $g$ down to the ultimate precision bound. The appropriate control is such that the filter function $F_{t}(\omega)=F_{t}$ is flat in the domain of $S(\omega)$, leading to an attenuation factor $\mathcal{J}=F_{t} g^{2}$ that is independent of the noise spectrum. For free evolution, this limit holds when the interval is much shorter than the correlation time $\tau_{c}$ of the environment, so that the qubit probe evolves freely. In Appendix D, we derive the optimal condition for attaining the ultimate estimation bound. This condition can be fulfilled only if $\tau_{c}$ is sufficiently large.

To overcome this limitation, we may instead realize such a filter at times larger than $\tau_{c}$ via repeated cycles of free evolution interlaced with stroboscopic projections of the qubit probe in the basis of $| \pm\rangle$, i.e., by quantum nondemolition (QND) measurements at a rate that is high enough to conform to the quantum Zeno regime [52]. The filter function then describes spectral broadening of the $| \pm\rangle$ eigenvalues far beyond the width of $S(\omega)$ [Fig. 1(b)]. The attenuation factor is then $[15,17,24]$ (Appendix D)

$$
\mathcal{J}_{\text {Zeno }}(g, t)=\frac{g^{2} t^{2}}{2 N}, \quad \text { if } \frac{t}{N} \ll \tau_{c},
$$

obtained for $N$ QND measurements during the total control time $t$. The advantage of this regime associated with the quantum Zeno effect (QZE) is that it requires $(t / N) \ll \tau_{c}$, rather than the total time $t$, to be less than $\tau_{c}$. Since the outcomes of these measurements need not be read out but rather used to guide the evolution, they may be emulated by impulsive noise-induced dephasing of the qubit probe that has the same effect as a projection on the probe evolution $[17,24,68]$. This QZE regime has been already exploited experimentally to determine the coupling strengths of complex spin networks [24].

Under the quantum Zeno condition of (15), the error estimation bound (11) is achieved when the total control time [after which $\sigma_{x}$ is measured and read out—see (5)] is chosen to have the optimal value

$$
t_{\text {Zeno }}^{\mathrm{opt}}=\frac{\sqrt{2 N \mathcal{J}_{0}}}{g}, \quad \text { provided that } N \gg \frac{2 \mathcal{J}_{0}}{g^{2} \tau_{c}^{2}} \text {. }
$$

This equation (further discussed in Appendix D) constitutes the main condition on an optimal filter designed to estimate $g$.

\section{Estimating the correlation time $\tau_{c}$}

To achieve the ultimate precision bound (11) for the estimation of $\tau_{c}$ of noise spectra (13) and (14), the control on the probe should generate a filter $F_{t}(\omega)$ that overlaps only with the power-law portion of $S_{\beta(s)}\left(\tau_{c}, \omega\right) \propto \tau_{c}^{\mp \alpha} \omega^{\mp(\alpha \pm 1)}$, as shown in Fig. 1(c). By contrast, FID of the probe coherence generates a filter (sinc) function centered at zero frequency, thus preventing the bound in Eq. (11) from being reached.

While various controls may allow the best estimation according to Eq. (11), we here analytically study the conditions for achieving the ultimate bound under standard CPMG sequences of $\pi$ pulses [69] or under continuouswave driving $(\mathrm{CW})$. In control sequences of equidistant pulses $N \gg 1$, the filter function $F_{t}(\omega)$ converges to a sum of delta functions (narrow-band filters) centered at the harmonics of the inverse CPMG time period, while for $\mathrm{CW}$ there is a single frequency component $[17,23,30]$ (Appendix D). In the following, we use these filter functions to analytically infer the required total control time $t$ and $N$ that allow the bound to be attained for the two classes of power-law spectra in Eqs. (13) and (14) (see Appendix D for details).

(i) For generalized Ornstein-Uhlenbeck spectra $S_{\beta}(\omega)$, only high frequencies must be probed by the dynamical-control filter. To this end, the intervals between pulses or refocusing periods must obey $(t / N) \ll \tau_{c}$. Then, $\mathcal{J}_{\beta} \propto \tau_{c}^{-(\beta-1)}$ satisfies the equality in (11) and the minimal relative error attains the ultimate bound, provided that the total control time is chosen to have the optimal value

$$
t_{\beta}^{\mathrm{opt}}=\tau_{c} \sqrt[\beta+1]{\frac{N^{\beta} \mathcal{J}_{0}}{c_{\beta} g^{2} \tau_{c}^{2}}},
$$


where $c_{\beta}$ is a constant depending on the control sequence. The bound can be attained only if $N$ is sufficiently large to satisfy $t_{\beta}^{\mathrm{opt}}, \tau_{c} \gg\left(t_{\beta}^{\mathrm{opt}} / N\right)$ and overlaps only with the power-law tail, as shown in Fig. 1(c). By contrast, FID fails this condition, since its filter mainly overlaps with $\omega \approx 0$ [Fig. 1(c)], causing a larger error in the estimation, as shown in Fig. 2(b).

(ii) For generalized Ohmic spectra $S_{s}$, the filter should overlap only with the noise spectrum at a frequency lower than the cutoff, $0<\omega<\omega_{c}$, avoiding any overlap at $\omega_{c}$. Then, $\mathcal{J}_{s} \propto \tau_{c}^{s+1}$ satisfies the equality in (11) and the minimal relative error attains the ultimate bound when the measurement of $\sigma_{x}$ is performed at the optimal total control time

$$
t_{s}^{\mathrm{opt}}=\tau_{c} \sqrt[s-1]{\frac{c_{s} g^{2} \tau_{c}^{2} N^{s}}{\mathcal{J}_{0}}}
$$

Here $\left(t_{s}^{\mathrm{opt}} / N\right)>\tau_{c}$ and $N \gg 1$ ensure the filter is narrow band with a negligible tail at $\omega_{c}$, as opposed to its FID counterpart that causes a much larger estimation error [see Fig. 2(b)].

Equations (17) and (18) thus constitute our main condition on the optimal filter design for estimating $\tau_{c}$, including the restrictions for $N$ and the filter function shapes.

\section{Power-law estimation}

One can optimally estimate the exponent $\beta$ or $s$ that governs the bath spectrum by maximizing the QFI (8) for the estimation of $x_{B}=\gamma=\beta+1=1-s$ (Appendix D). This maximization leads to the ultimate precision bound

$\varepsilon(\gamma, t) \geq \frac{1}{\gamma \sqrt{N_{m} \mathcal{F}_{\mathcal{Q}}(\gamma, t)}} \geq \frac{\sqrt{1-e^{-2 \mathcal{J}_{1}}}}{\sqrt{N_{m}} \mathcal{J}_{1}\left|\ln \left(\mathcal{J}_{1}\right)\right| e^{-\mathcal{J}_{1}}}=\frac{\varepsilon_{1}}{\sqrt{N_{m}}}$,

with $\mathcal{J}_{1}=e^{-2.246} \approx 0.106$ and $\varepsilon_{1} \approx 2.04$. The bound is achieved when the qubit probes only the power-law regime of noise spectra (13) and (14). Provided that the CPMG or $\mathrm{CW}$ dynamical control probes the power-law region, the bound is attained when the qubit probe is measured at

$$
t_{\mathrm{opt}}=T_{2} \sqrt[\gamma]{\mathcal{J}_{1}},
$$

where $\mathcal{J}(\gamma, t)=\left(t / T_{2}\right)^{\gamma}$ and $T_{2}$ is the dephasing time (that depends on the applied control).

\section{REAL-TIME ESTIMATION PROTOCOL}

The predicted optimal time $t^{\text {opt }}$ for performing a measurement following the dynamical control or FID of the quantum-probe coherence explicitly depends on the unknown parameter $x_{B}$ to be estimated [see Eqs. (16), (17), (18), and (20)]. In practice, one may bypass this difficulty by estimating $x_{B}$ and simultaneously finding the optimal time to monitor the probe via an efficient real-time estimation protocol $[60,61,63]$. We illustrate here the implementation of this protocol and show that it attains the predicted ultimate bound (11) if the qubit probe is optimally controlled. The protocol is based on a Bayesian estimator and an online experimental learning design that maximizes the information gain, similar to the one that was recently implemented for improving the suppression of decoherence in quantum dots [62], adaptive real-time pulse shaping [70], $a b$ initio optical phase estimation [71], or stabilizing Rabi oscillations using quantum feedback [72]. Its stages are as follows.

(i) We initially guess a probability distribution $p\left(x_{B}\right)$ for the unknown parameter $x_{B}$ that represents our a priori knowledge of the parameter to be estimated. Physically, we should have $x_{B}>0$, and therefore one can assume a flat distribution of $p\left(x_{B}\right)$, $x_{B} \in\left(x_{B}^{\min }, x_{B}^{\max }\right]$ that contains the true value $x_{B}^{\text {true }}$.

(ii) We then determine the best time $t_{m}$ to perform a measurement for maximizing the information gain about $x_{B}$ which is defined by the information entropy $U\left(t_{m}\right)=\max _{t}\{U(t)\}$ (Appendix E), which depends on $p\left(x_{B}\right)$ and the likelihood function $p\left(d \mid x_{B}, t\right)$ of Eq. (5) that determines the conditional probability to obtain the possible outcome data of the measurement $d=\{+,-\}$.

(iii) We next perform a measurement of $\sigma_{x}$ of the qubitprobe state, initialized in the state $|+\rangle$, at the optimal time $t_{m}$, obtaining the outcome data $d$ with probability $p\left(d \mid x_{B}^{\text {true }}, t\right)$. According to the obtained data, we update our knowledge of $x_{B}$ by the Bayesian rule

$$
p^{\text {new }}\left(x_{B}\right) \equiv p\left(x_{B} \mid d, t_{m}\right)=\frac{p\left(d \mid x_{B}, t_{m}\right) p\left(x_{B}\right)}{p\left(d \mid t_{m}\right)},
$$

where $p(d \mid t)$ is a normalization factor for integration over $x_{B}$.

The estimation of $x_{B}$ improves upon iteratively repeating this three-stage process $N_{m}$ times, $N_{m}$ standing for the number of measurements. When an adequate control is chosen, the probability distribution $p\left(x_{B}\right)$ converges to a narrow peak around $x_{B}^{\text {true }}$. By contrast, the convergence under free evolution can be very poor.

Figure 3 presents a simulated experiment of this iterative process for the estimation of $g$ and $\tau_{c}$ of the environmental noise by a NVC spin probe in two types of diamond samples whose intrinsic environmental characteristics require dynamical control to attain the ultimate bound (11). (a) We simulate the estimation of $\tau_{c}$ for the NVC probe evolving freely (undergoing FID), as compared to it being nearly optimally controlled by a CPMG sequence with $N=8$ subject to an Ornstein-Uhlenbeck process characterized by $G_{\beta=2}\left(\tau_{c}^{\text {true }}=10 \mu \mathrm{s}, \omega\right)$ with $g=1 \mathrm{MHz}$ (consistent with the high-pressure 

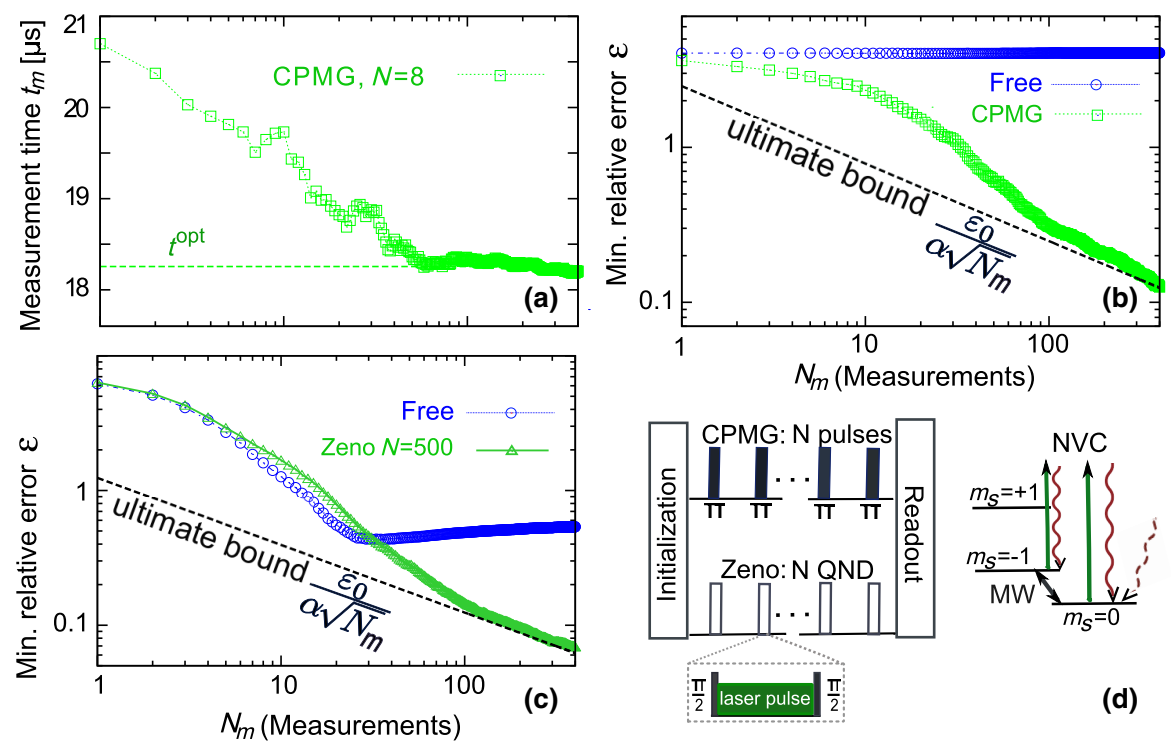

FIG. 3. Simulation of an experimental real-time adaptive-estimation protocol for realistic conditions with a NVC spin probe. (a),(b) Convergence of the real-time adaptive-estimation protocol to the theoretically predicted values for estimating $\tau_{c}$. Free evolution of the probe (blue circles) is contrasted with that of a dynamically controlled probe under a CPMG (green square) sequence with $N=8$ in the presence of an Ornstein-Uhlenbeck process with Lorentzian spectrum $G_{\beta=2}\left(\tau_{c}^{\text {true }}=10 \mu \mathrm{s}, \omega\right)$, with $g=1 \mathrm{MHz}$ consistently with the spectral density of a HPHT diamond sample determined in Ref. [54]. The simulated curves derived from exact analytical results of Eq. (4) [11] are averaged over 600 realizations. In (a), the optimal measurement time $t_{m}$ as a function of $N_{m}$ converges to the value $t^{\mathrm{opt}}$ for the CPMG case. Similar curves converging to the corresponding $t^{\mathrm{opt}}$ are observed for other controls and free evolution. In (b), the minimal relative error $\varepsilon\left(\tau_{c}, t^{\text {opt }}\right)$ converges to the (Cramer-Rao) bound. Under free evolution, the regime where $\varepsilon \propto\left(1 / \sqrt{N_{m}}\right)$ is attained for $N_{m} \gg 100$. The ultimate bound $\left(\varepsilon_{0} / \sqrt{N_{m}}\right.$ dashed line, $\alpha=\beta-1$ ) is attained only by optimal control. (c) Convergence to the minimal relative error $\varepsilon\left(g, t_{\mathrm{opt}}\right)$ to the (Cramer-Rao) bound for estimating $g$ by $N=500$ consecutive projective measurements in the Zeno regime (green triangle) compared to the estimation under free evolution (blue circle). In this case, $G_{\beta=2}(g=0.03 \mathrm{MHz}, \omega)$, with $\tau_{c}=10 \mu \mathrm{s}$, consistently with the spectral density of a ${ }^{12} \mathrm{C}$ diamond sample determined in Ref. [54]. Here too the $u l t i m a t e ~ b o u n d\left(\varepsilon_{0} / 2 \sqrt{N_{m}}\right.$ dashed line, $\alpha=2$ ) is attained only by optimal control. (d) Proposed scheme for using a NVC as a qubit probe for its environment. The $m_{s}=0(|0\rangle)$ state is fully populated by laser irradiation (dashed curly arrow). Microwave (MW) pulses are selectively applied between the states with $m_{s}=0$ and $-1(|0\rangle$ and $|-1\rangle)$ of the electronic ground states to initialize the spin probe in a $|+\rangle=(1 / \sqrt{2})(|0\rangle+|-1\rangle)$ state and effect the $\pi$ pulse CPMG sequence for estimating $\tau_{c}$. For estimating $g$, projective measurements are emulated by combining MW $\pi / 2$ pulses on the $0 \leftrightarrow-1$ transition and laser-induced relaxation between the ground and exited electronic states that conserve the spin components (solid curly arrows). The readout is done at the end of the $N$-pulse sequence by detecting the laser-induced fluorescence signal.

high-temperature (HPHT) diamond sample data of Ref. [54]). Our simulated best-measurement timings $t_{m}$ converge to the theoretically predicted $t^{\text {opt }}$ that maximizes the QFI (8), as exemplified in Fig. 3(a) for CPMG control, where $t^{\mathrm{opt}} \approx 18.25 \mu \mathrm{s}$ is on time scales compatible with the accessible experimental times [54]. Concurrently, the minimum relative error converges to $\varepsilon\left(\tau_{c}, t_{\text {opt }}\right)$ predicted from the ultimate Cramer-Rao bound [Fig. 3(b)].

(b) In Fig. 3(c), we simulate the estimation of $g$ under similar conditions by a sequence of $N \gg 1$ QND measurements in the Zeno (QZE) regime. Since we need not read out the results of these measurements, they may be emulated by brief (stroboscopic) induced dephasings of the probe coherence that effectively interrupt the free (FID) evolution [15,73]. This protocol is experimentally implemented in Refs. $[24,68]$ by applying repetitive random magnetic-field gradients or random shifts of the effective probe frequency [74-76]. In addition, we may initialize the NVC probe in its electronic ground state and then induce dephasing in the $| \pm\rangle$ basis by $\pi / 2$ MW pulses separated by a laser pulse [Fig. 3(d)]. The laser pulse will induce a transition to the electronic excited state conserving the spin superposition state [77]. The subsequent radiative decay to the electronic ground state, which is longitudinally spin conserving [78], will effectively collapse the wave function (see Appendix F). In Fig. 3(c), we compare the results obtained following $N=500$ of such Zeno-like dephasings to the FID results. For the simulations, we assume them to be perfect, stroboscopic, projections of the spin state onto the $| \pm\rangle$ basis. The optimal intervals between such dephasings are found to be in this case $\left(t_{\text {opt }} / N\right) \approx 1.9 \mu \mathrm{s}$. We then find by this Zeno (QZE) method the convergence to the ultimate Cramer-Rao bound, where the spectral density $G_{\beta=2}(g=0.03 \mathrm{MHz}, \omega)$ with $\tau_{c}=10 \mu$ s is consistent 
with the spectral density determined in Ref. [54] for the ${ }^{12} \mathrm{C}$ diamond sample. This Zeno estimation procedure is performed without assuming the line shape of the spectral density. It is seen from Fig. 3(c) that the precision error eventually saturates for the free evolution (FID) at a precision that is limited by our lack of knowledge of the line shape. By contrast, this saturation can be overcome in the QZE regime discussed above.

The foregoing simulations thus confirm, under experimentally realistic conditions, our second major analytical result, whereby the ultimate theoretical bound (11) is indeed attainable under optimal control but hardly ever under free evolution.

\section{DISCUSSION}

We demonstrate that dynamical control of a quantum probe not only dramatically improves the quantum estimation of environmental parameters compared to the freeevolution (induction) decay of its coherence: It may be imperative to use such control, since FID may preclude their correct estimation. In particular, for generic types of noise spectra, as in generalized Ornstein-Uhlenbeck and Ohmic processes, the ultimate analytical bounds for the coupling strength and correlation-time estimation precision derived here can be achieved by optimizing the dynamical control on the probe. The optimal controls suitable for the estimation of $g, \tau_{c}$ (Fig. 1 and 3), or the power-law exponent are generally different, but the protocol is similar. Specifically, we demonstrate that the ultimate estimation bound for $g$ can be attained in the quantum Zeno regime without prior knowledge of the environmental spectraldensity shape. Once this probe-environment coupling is known, the environmental correlation time can be reliably estimated by standard dynamical-control sequences.

Experimental conditions typically require a minimized average error within the coherence time interval shown in Fig. 2(b), if this interval is much shorter than the combined postmeasurement readout and initialization time of the qubit. Under such conditions, an important implication of the present analysis is that it yields the optimal total time of the premeasurement dynamical control and thereby the number of pulses and the delay between them that need be applied to attain the best precision per measurement. However, in the (rarely encountered) opposite limit of fast readout and initialization, the overall error rate of the measurements is to be minimized. Then, a similar optimization can be performed by maximizing the Fisher information per unit time. If additional specific constraints are imposed by a particular experimental setup, e.g., limited power or estimation time, then further optimization is needed to find the optimal filter function. In such cases, the goal would be to approach as best we can the ultimate error bound under the given constraints.
A real-time adaptive-estimation protocol illustrates the ability to find the optimal time of dynamical control for achieving the predicted precision bounds, e.g., for a NVC in a diamond that acts as a qubit probe of the bulk or surface in optically detected NMR and MRI. Its aim here is to determine the noise spectrum generated by nuclear or electronic spins [6-8,43,44], as well as other sources of dephasing. In Fig. 3, we illustrate this protocol for estimating both $g$ and $\tau_{c}$ near a NVC used as a probe. The inferred correlation times of the noise fluctuations can be particularly helpful for studying molecular diffusion at the nanoscale, where the power-law tails of noise spectra allow the extraction of the diffusion rates and restriction lengths associated with pore structures and thereby characterizing biological processes $[11,42,66]$.

In other scenarios, power-law tails can also characterize charge diffusion in conducting crystals [59] or spin diffusion in complex spin networks $[23,24,58,79,80]$. Generalized Ohmic spectra may help understand the functioning of nanoscale electromechanical devices [81], as well as superconducting devices attached to conducting leads [82].

Several extensions of the outlined strategy will be further explored.

(a) If uncontrollable noise sources are present, such as an intrinsic $T_{2}$ decoherence due to a white-noise (Markovian) process or due to pulse imperfections, we may have to resort to more elaborate controls, nicknamed by us "selective dynamical recoupling" (SDR), which we show to allow selective probing of the targeted noise source $[10,11]$. SDR was already implemented to selectively monitor diffusion processes characterized by an Ornstein-Uhlenbeck spectral density, so as to determine the probe-environment coupling strengths $[10,64]$ and the environmental correlation time, as well as diffusion restriction lengths $[11,42,66]$ in systems of biological and chemical interest. We envisage that by incorporating SDR within the present optimized estimation strategy one may eliminate Markovian (intrinsic- $T_{2}$ and pulseerror) effects and allow a clean parameter estimation of the targeted noise spectrum.

(b) A qubit probe may be replaced by an entangled $n$-particle probe that may yield a different error bound $[53,83,84]$. The estimation strategy remains similar, but the dynamical control has to be adapted to a multipartite scenario, based on the approach of Refs. $[26,85,86]$.

(c) The present strategy, whereby environmental parameters are estimated one by one, provides the ultimate precision bounds that can be attained for estimating a single parameter, assuming that the remaining parameters are known with much better accuracy. Therefore, these bounds are also valid for more elaborate multiparameter estimation strategies based on a quantum Fisher information matrix [47,50,87], although these 
bounds may not be tight in the latter case. Still, the single-parameter estimation expounded here may be superseded by a multiparameter optimized estimation per measurement.

In conclusion, we analytically set the error bounds on environmental-parameter estimation and demonstrate the ability to extract such environmental information with maximal accuracy by the least number of measurements possible upon invoking dynamical control. This general result for a qubit probe weakly coupled to its environment opens the door to the development of an important diagnostic tool of environmental processes by quantum probes.

\section{ACKNOWLEDGMENTS}

We thank L. Frydman and N. Shemesh for fruitful discussions. G. A. A. acknowledges the support of the European Commission under the Marie Curie IntraEuropean Fellowship for Career Development Grant No. PIEF-GA-2012-328605. G. K. acknowledges the ISF support under the Bikura (Prime) grant.

\section{APPENDIX A: ANAMNESIS: FILTER FUNCTION DERIVATION FOR QUBIT PROBE UNDER DEPHASING (FOLLOWING REFS. [15-17,26,27])}

The Hamiltonian of a single spin probe interacting with a bath that produces pure dephasing is

$$
H=H_{S}(t)+H_{B}+H_{\mathrm{SB}}
$$

with

$$
H_{S}=\omega_{0} \sigma_{z}+f_{0}(t) \sigma_{x}, \quad H_{\mathrm{SB}}=S \otimes B=\sigma_{z} g B,
$$

where $f_{0}(t)=f(t) e^{-i \omega_{0} t}+$ c.c. is the dynamical control applied to the qubit, $B$ and $S$ are the operators of the bath and the system ( $\sigma_{z}$ for pure dephasing), respectively, and $g$ is their coupling strength.

One can derive the non-Markovian master equation for the density matrix of the system, $\rho_{S}(t)$ in the interaction picture, which in the Born approximation one assumes a weak coupling $g$ such that the influence of the qubit probe on the environment is small (usually called the weak-coupling approximation). In this approximation, the density matrix of the environment $\rho_{B}$ is only negligibly affected by the interaction with the qubit probe, and the state of the total system at time $t$ is allowed to be expressed as $\rho(t) \approx \rho_{S}(t) \otimes \rho_{B}$ [67]. The resulting non-Markovian master equation is then given by $[15-17,88,89]$

$\dot{\rho}_{S}\left(x_{B}, t\right)=\int_{0}^{t} d t^{\prime}\left\{g^{2} \Phi\left(x_{B}, t-t^{\prime}\right)\left[S\left(t^{\prime}\right), S(t) \rho_{S}(t)\right]+\right.$ H.c. $\}$, where $\Phi\left(x_{B}, t^{\prime}-t^{\prime \prime}\right)=\operatorname{Tr}_{B}\left\{B\left(t^{\prime}-t^{\prime \prime}\right) B(0) \rho_{B}(0)\right\}$ are the bath correlation functions, $x_{B}$ is a parameter that characterizes the environment, and

$$
\begin{aligned}
& S(t)=U_{S}^{\dagger}(t) S U_{S}(t) U_{S}(t)=\mathcal{T} e^{-i \int_{0}^{t} d t^{\prime} H_{S}\left(t^{\prime}\right)}, \\
& B(t)=U_{B}^{\dagger}(t) B U_{B}(t), \quad U_{B}(t)=e^{-i H_{B} t} .
\end{aligned}
$$

Since we are interested in describing the dephasing, the phase due to the unperturbed energy difference $\hbar \omega_{0}$ is irrelevant. To eliminate this dependence, we move to the rotating frame, where the time-dependent basis consists of

$$
| \pm\rangle=\frac{1}{\sqrt{2}}\left(e^{-i \omega_{0} t}|\uparrow\rangle+|\downarrow\rangle\right)
$$

In this basis, the system Hamiltonian becomes

$$
\tilde{H}_{S}=f(t) \tilde{\sigma}_{z}, \quad \tilde{\sigma}_{z}=|+\rangle\langle+|-|-\rangle\langle-| .
$$

We then rewrite Eqs. (4) as

$$
\begin{aligned}
U_{S}(t) & =e^{-i \int_{0}^{t}\left(f\left(t^{\prime}\right) / 2\right) d t}|+\rangle\left\langle+\left|+e^{i \int_{0}^{t}\left(f\left(t^{\prime}\right) / 2\right) d t}\right|-\right\rangle\langle-| \\
S(t) & =e^{i \int_{0}^{t} f\left(t^{\prime}\right) d t}|+\rangle\left\langle-\left|+e^{i \int_{0}^{t} f\left(t^{\prime}\right) d t}\right|-\right\rangle\langle+| .
\end{aligned}
$$

Plugging Eqs. (A7) into the master equation Eq. (A3), we arrive at $[15-17,88,89]$

$$
\frac{d}{d t}\left(\Delta \rho_{S, \pm}\right)=-\frac{d \mathcal{J}}{d t} \Delta \rho_{S, \pm} .
$$

Here $\Delta \rho_{S, \pm}=\rho_{S,++}-\rho_{S,--}$ is the spin coherence in the basis $|\uparrow\rangle,|\downarrow\rangle$ at time $t$ :

$$
\left\langle\sigma_{x}(t)\right\rangle=\left\langle\tilde{\sigma}_{z}(t)\right\rangle=\Delta \rho_{S, \pm}=e^{-\mathcal{J}\left(x_{B}, t\right)}
$$

characterized by the attenuation factor $[15-17,88,89]$

$$
\mathcal{J}\left(x_{B}, t\right)=\operatorname{Re} \int_{0}^{t} d t^{\prime} \int_{0}^{t^{\prime}} d t^{\prime \prime} g^{2} \Phi\left(x_{B}, t^{\prime}-t^{\prime \prime}\right) \Omega\left(t^{\prime}\right) \Omega^{*}\left(t^{\prime \prime}\right),
$$

where we have set

$$
\Omega(t)=e^{i \int_{0}^{t} f\left(t^{\prime}\right) d t} .
$$

This attenuation factor can be cast in the spectral form

$$
\mathcal{J}\left(x_{B}, t\right)=\int_{-\infty}^{\infty} d \omega F_{t}(\omega) G\left(x_{B}, \omega\right)
$$

where

$$
G\left(x_{B}, \omega\right)=\frac{1}{2 \pi} \int_{-\infty}^{\infty} d t g^{2} \Phi\left(x_{B}, t\right) e^{i \omega t}
$$


is the bath-coupling spectrum and

$$
F_{t}(\omega)=\frac{1}{2 \pi}\left|\int_{0}^{t} d t^{\prime} \Omega\left(t^{\prime}\right) e^{i \omega t^{\prime}}\right|^{2}
$$

is the filter function which is determined by the dynamical control of the qubit probe.

We stress that $\Omega(t)$ can have an arbitrary temporal shape, as opposed to the restrictions on its (inherently pulsed) shape in the dynamical decoupling method $[13,14,18,29]$.

\section{APPENDIX B: QUANTUM FISHER INFORMATION CONCERNING A SINGLE PARAMETER OF THE ENVIRONMENT}

The QFI concerning a single parameter $x_{B}$ of the environment (bath) for the qubit state is [46-49]

$$
\begin{aligned}
\mathcal{F}_{\mathcal{Q}}= & \frac{1}{p_{+}}\left(\frac{\partial p_{+}}{\partial x_{B}}\right)^{2}+\frac{1}{p_{-}}\left(\frac{\partial p_{-}}{\partial x_{B}}\right)^{2} \\
& +2 \frac{\left(p_{+}-p_{-}\right)^{2}}{p_{+}+p_{-}}\left(\mid\left\langle p_{-}\left|\frac{\partial\left|p_{+}\right\rangle}{\partial x_{B}}\right|^{2}+\right|\left\langle p_{+}\left|\frac{\partial\left|p_{-}\right\rangle}{\partial x_{B}}\right|^{2}\right),\right.
\end{aligned}
$$

where

$$
\begin{aligned}
p_{ \pm}\left(x_{B} t\right) & \equiv p\left( \pm \mid x_{B}, t\right)=\frac{1}{2}\left(1 \pm e^{-\mathcal{J}\left(x_{B}, t\right)}\right), \\
\left|p_{ \pm}\right\rangle & =\frac{1}{\sqrt{2}}\left(e^{-i \omega_{0} t}|\uparrow\rangle \pm|\downarrow\rangle\right)
\end{aligned}
$$

are the eigenvalues and eigenvectors of the spin-probe density matrix $[17,89]$. A measurement is said to be optimal when the QFI $\mathcal{F}_{\mathcal{Q}}$ coincides with its classical counterpart [46-49]. This is the case here under pure dephasing, when the last term in (B1) is null due to $\partial\left|p_{+}\right\rangle / \partial x_{B}=0$. Then the optimal measurement is effected by projections onto the eigenstates $\left|p_{ \pm}\right\rangle$of $\sigma_{x}$ in the rotating frame

$$
\begin{aligned}
\left|p_{ \pm}\right\rangle\left\langle p_{ \pm}\right| & =\frac{1}{2} e^{-i\left(\omega_{0} / 2\right) \sigma_{z}}| \pm\rangle\langle \pm| e^{i\left(\omega_{0} / 2\right) \sigma_{z}}, \\
| \pm\rangle & =\frac{1}{\sqrt{2}}(|\uparrow\rangle \pm|\downarrow\rangle) .
\end{aligned}
$$

Correspondingly, Eq. (B1) becomes

$$
\mathcal{F}_{\mathcal{Q}}\left(x_{B}, t\right)=\frac{e^{-2 \mathcal{J}\left(x_{B}, t\right)}}{1-e^{-2 \mathcal{J}\left(x_{B}, t\right)}}\left(\frac{\partial \mathcal{J}\left(x_{B}, t\right)}{\partial x_{B}}\right)^{2},
$$

if the initial probe state is $|+\rangle$. An arbitrary initial state, $[\cos (\theta)|\uparrow\rangle+i \sin (\theta)|\downarrow\rangle], 0<\theta<(\pi / 2)$, leads to $\mathcal{F}_{\mathcal{Q}}\left(x_{B}, t\right) \propto$ $\sin ^{2}(2 \theta)$ [48]. Therefore, the optimal initial state leading to the maximal QFI is obtained for $\theta=(\pi / 4)$, $|+\rangle=(1 / \sqrt{2})(|\uparrow\rangle+|\downarrow\rangle)$, thus proving Eq. (11) in the main text.
APPENDIX C: DERIVATION OF THE ULTIMATE PRECISION BOUND

A broad class of practically relevant environmental noise processes cause the attenuation factor to have a power-law functional dependence in $x_{B}$ with exponent $\alpha$, where the derivative of $\mathcal{J}\left(x_{B}, t\right)$ with respect to $x_{B}$ satisfies

$$
\left|\frac{\partial \mathcal{J}\left(x_{B}, t\right)}{\partial x_{B}}\right| \leq \frac{\alpha \mathcal{J}\left(x_{B}, t\right)}{x_{B}} .
$$

The QFI then conforms to the inequality

$$
\mathcal{F}_{\mathcal{Q}}\left(x_{B}, t\right) \leq \frac{e^{-2 \mathcal{J}\left(x_{B}, t\right)}}{1-e^{-2 \mathcal{J}\left(x_{B}, t\right)}} \frac{\alpha^{2} \mathcal{J}\left(x_{B}, t\right)^{2}}{x_{B}^{2}} .
$$

The equality is obtained when the attenuation factor is a homogeneous function of degree $\alpha$, i.e., strictly obeys a power law. The maximum of the QFI, regardless of the kind of control applied, is then obtained when $\left|\partial \mathcal{J}\left(x_{B}, t\right) / \partial x_{B}\right|=$ $\left(\alpha \mathcal{J}\left(x_{B}, t\right) / x_{B}\right)$ and $\mathcal{J}\left(x_{B}, t\right)=\mathcal{J}_{0}=1+\frac{1}{2} W\left(-2 e^{-2}\right) \approx 0.8$, where $W(z)$ is the Lambert function which by definition satisfies $z=W(z) e^{W(z)}$ for any complex number $z$. When the control is such that the equality in $(\mathrm{C} 2)$ is satisfied, then the optimal total control time at which the measurement should be done, $t_{\text {opt }}$, is such that $\mathcal{J}\left(x_{B}, t_{\text {opt }}\right)=\mathcal{J}_{0}$.

Under this condition, the resulting ultimate bound for the relative error in the estimation of $x_{B}$, which holds for arbitrary control on the probe, is

$\varepsilon\left(x_{B}, t\right) \geq \frac{1}{x_{B} \sqrt{N_{m} \mathcal{F}_{\mathcal{Q}}\left(x_{B}, t\right)}} \geq \frac{\sqrt{1-e^{-2 \mathcal{J}_{0}}}}{\alpha \sqrt{N_{m}} \mathcal{J}_{0} e^{-\mathcal{J}_{0}}}=\frac{\varepsilon_{0}}{\alpha \sqrt{N_{m}}}$,

with $\left.\varepsilon_{0}=\sqrt{2 /\left\{-W\left(-2 e^{-2}\right)\left[1+\frac{1}{2} W\left(-2 e^{-2}\right)\right]\right.}\right\} \approx 2.48$.

\section{Precision bounds for the key parameters $g$ and $\tau_{c}$ \\ $a$. The probe-bath interaction strength $g$}

The attenuation factor is a homogeneous function of degree $\alpha=2$ in the effective probe-bath interaction strength $g$, where $G\left(\tau_{c}, \omega\right)=g^{2} S(\omega)$ with $S(\omega)$ the normalized spectral density of the environmental noise. Therefore, Eqs. (C1) and (C2) are satisfied, and the minimal relative error in the estimation of $g$ is obtained by measuring at the time $t_{\text {opt }}$, such that $\mathcal{J}\left(g, t_{\text {opt }}\right)=\mathcal{J}_{0}$, if $S(\omega)$ is known.

\section{b. The correlation time $\boldsymbol{\tau}_{\boldsymbol{c}}$}

The derivative term in the QFI of Eq. (B4) depends on the derivative of the bath coupling spectrum (spectral density) with respect to the correlation time $\partial G / \partial \tau_{c}$, through

$$
\frac{\partial \mathcal{J}\left(\tau_{c}, t\right)}{\partial \tau_{c}}=\int_{-\infty}^{\infty} d \omega F_{t}(\omega) \frac{\partial G\left(\tau_{c}, \omega\right)}{\partial \tau_{c}} .
$$


Spectral densities of the baths characterized by $G_{\beta(s)}\left(\tau_{c}, \omega\right)=g^{2} S(\omega)$ [defined in the main text, Eqs. (13) and (14)] satisfy $\left|\partial S_{\beta(s)} / \partial \tau_{c}\right| \leq\left(\alpha S_{\beta(s)} / \tau_{c}\right)$. Specifically,

$$
\begin{aligned}
\left|\frac{\partial G_{\beta}}{\partial \tau_{c}}\right| & =\left|\frac{\partial}{\partial \tau_{c}}\left(\frac{\mathcal{A}_{\beta} g^{2} \tau_{c}}{1+\omega^{\beta} \tau_{c}^{\beta}}\right)\right| \\
& =\left|\frac{\mathcal{A}_{\beta} g^{2}}{1+\tau_{c}^{\beta} \omega^{\beta}}\left(1-\frac{\beta \tau_{c}^{\beta} \omega^{\beta}}{1+\tau_{c}^{\beta} \omega^{\beta}}\right)\right| \\
& \leq \frac{\mathcal{A}_{\beta} g^{2}(\beta-1)}{\left(1+\tau_{c}^{\beta} \omega^{\beta}\right)}=\frac{(\beta-1) G_{\beta}}{\tau_{c}},
\end{aligned}
$$

where the equality is attained when the spectral density function behaves as a homogeneous function of degree $\alpha=\beta-1, G_{\beta} \propto \tau_{c}^{-(\beta-1)} \omega^{\beta}$; and

$$
\frac{\partial G_{s}}{\partial \tau_{c}}=\frac{\partial}{\partial \tau_{c}}\left[(s+1) \tau_{c}^{s+1} \omega^{s}\right]=\frac{(s+1)}{\tau_{c}} G_{s}
$$

since $G_{s}$ is a homogeneous function of degree $\alpha=s+1$ upon neglecting the cutoff region.

Using Eqs. (C5) and (C6) to bound Eq. (C4), we find

$$
\begin{aligned}
\left|\frac{\partial \mathcal{J}\left(\tau_{c}, t\right)}{\partial \tau_{c}}\right| & =\int_{-\infty}^{\infty} d \omega F_{t}(\omega)\left|\frac{\partial G\left(\tau_{c}, \omega\right)}{\partial \tau_{c}}\right| \\
& \leq \frac{\alpha}{\tau_{c}} \int_{-\infty}^{\infty} d \omega F_{t}(\omega) G\left(\tau_{c}, \omega\right) \\
& =\frac{\alpha \mathcal{J}\left(\tau_{c}, t\right)}{\tau_{c}} .
\end{aligned}
$$

This leads to the tight bound for the Fisher information (C2) and, therefore, for the minimal error in the estimation (C3) which holds for arbitrary control on the spin probe. The ultimate bound in the precision (C3) is attained when the optimal total control time at which the measurement should be done, $t_{\text {opt }}$, is such that $\mathcal{J}\left(\tau_{c}, t_{\text {opt }}\right)=\mathcal{J}_{0}$.

\section{APPENDIX D: ATTAINMENT OF THE ULTIMATE BOUND}

\section{Attainment of the ultimate precision bound under optimal dynamical control in the estimation of key parameters}

\section{a. Probe-bath interaction strength $g$}

The attenuation factor is a homogeneous function of degree $\alpha=2$ in the probe-bath interaction strength $g$. Therefore, Eqs. (C1) and (C2) are satisfied, and the minimal relative error in the estimation of $g$ is attained by measuring at a time $t_{\text {opt }}$ such that $\mathcal{J}\left(g, t_{\text {opt }}\right)=\mathcal{J}_{0}$ if $S(\omega)$ is known.

If $S(\omega)$ is not known, then some constraints apply for $t_{\text {opt }}$. Considering that the attenuation factor is given by Eq. (A12), then, if the filter function $F_{t}(\omega)$ is much wider than $G(g, \omega)=g^{2} S(\omega)$, it can be considered as a constant
$F_{t}$ in the integral (A12). The attenuation factor following the integration is then $g^{2} F_{t}$, where we have used the normalization property of the spectral density $S(\omega)$. This limit holds when the interval is shorter than the correlation time $\tau_{c}$ of the environment, so that the qubit probe evolves freely, yielding [15-17,88,89]

$$
F_{t}^{\mathrm{free}}(\omega)=\frac{t^{2}}{2} \operatorname{sinc}^{2}\left(\frac{\omega t}{2}\right) \approx \frac{t^{2}}{2} \quad \text { if } t \ll \tau_{c},
$$

and

$$
\mathcal{J}^{\text {free }}(g, t) \approx \frac{1}{2} g^{2} t^{2}
$$

Then, the bound in the estimation error (C3) is achieved when

$$
t_{\mathrm{opt}}^{\text {free }}=\frac{\sqrt{2 \mathcal{J}_{0}}}{g}, \quad \text { provided } t_{\mathrm{opt}}^{\text {free }}=\sqrt{\frac{2 \mathcal{J}_{0}}{g^{2}}} \ll \tau_{c} .
$$

This condition can be fulfilled only for large enough $\tau_{c}$. To overcome this limitation, we may exploit dynamical control by means of frequent, stroboscopic QND measurements of the qubit probe. If $N$ QND unread measurements are performed during a total time $t$, the dynamics conforms to the Zeno regime with an attenuation factor $[15-17,88,89]$

$$
\mathcal{J}^{\text {Zeno }}(g, t)=\frac{g^{2} t^{2}}{2 N} \quad \text { if } \frac{t}{N} \ll \tau_{c} .
$$

Then, the condition for attaining the bound in the estimation error (C3) is relaxed by $1 / \sqrt{N}$, as

$t_{\mathrm{opt}}^{\mathrm{Zeno}}=\frac{\sqrt{2 N \mathcal{J}_{0}}}{g}, \quad$ provided $\frac{t_{\mathrm{opt}}^{\mathrm{Zeno}}}{N}=\sqrt{\frac{2 \mathcal{J}_{0}}{g^{2} N}} \ll \tau_{c}$.

The latter condition can be attained for

$$
N \gg \frac{2 \mathcal{J}_{0}}{g^{2} \tau_{c}^{2}}
$$

In order to ensure that Eq. (D6) is satisfied, suffice it that the product $g \tau_{c}$ be roughly estimated, by observing the change in the decay law from the anti-Zeno or Fermi golden rule to the QZE regime as $N$ increases [90].

\section{b. Correlation time $\tau_{c}$}

Control of the spin probe by $N$-pulse CPMG sequences or $N$ cycles of $\mathrm{CW}$ driving leads to a filter function $F_{t}(\omega)$ that converges to a Fourier series described by a sum of delta functions (band narrow filters) centered at the harmonics of the inverse cycle time, $k \omega_{0}=(\pi k N / t)$, $k \in \mathbb{N}$, provided the total control time exceeds the interval between the pulses $t \gg(t / N)$. Under these conditions, the attenuation factor (A12) becomes [23,30] 


$$
\mathcal{J}\left(\tau_{c}, t\right)=g^{2} \sum_{k=1}^{\infty} F_{t}\left(k \omega_{0}\right) G\left(\tau_{c}, k \omega_{0}\right)
$$

In what follows, we infer the conditions for attaining the bound from the attenuation factor for the bath spectra considered in the main text.

(i) For generalized Ornstein-Uhlenbeck spectra $G_{\beta}$, one condition to achieve the bound of Eq. (C3) is that the filter overlap only with the power-law tail spectra. This is ensured when the first harmonic of the filter is already in this spectral region, which amounts to $(t / \pi N) \ll \tau_{c}$. The attenuation factor (D7) then becomes

$\mathcal{J}_{\beta}\left(\tau_{c}, t\right)=g^{2} \sum_{k=1}^{\infty} F_{t}\left(\frac{\pi k N \tau_{c}}{t}\right) \frac{\mathcal{A}_{\beta} \tau_{c}}{\left(\frac{\pi k N \tau_{c}}{t}\right)^{\beta}}=\frac{c_{\beta} g^{2} t^{\beta+1}}{N^{\beta} \tau_{c}^{\beta-1}}$,

with $\quad \mathcal{A}_{\beta}=(\beta / 2 \pi) \sin (\pi / \beta) \quad$ and $\quad c_{\beta}=$ $\left(\beta / 2 \pi^{\beta}\right) \sin (\pi / \beta)$ for $\mathrm{CW}$ (only the first harmonic $k=1$ gives non-null terms) and $c_{\beta}=[(\zeta(\beta+$ $\left.\left.2)\left(4-2^{-\beta}\right) \beta\right) / \pi^{2 \beta}\right] \sin (\pi / \beta)$ for CPMG (only odd $k$ gives non-null terms), where $\zeta$ is the zeta function defined for $\operatorname{Re}(z)>1$ as $\zeta(z)=\sum_{i=1}^{\infty}\left(1 / i^{z}\right)$. Both constants have similar values $c_{\beta}^{\mathrm{CW}} \approx c_{\beta}^{\mathrm{CPMG}}$.

Since $\mathcal{J}_{\beta}$ satisfies the equality in Eq. (C2), the ultimate bound (C3) is achieved when $\mathcal{J}_{\beta}\left(\tau_{c}, t_{\text {opt }}\right)=$ $\mathcal{J}_{0}$. This yields

$$
t_{\beta}^{\mathrm{opt}}=\tau_{c} \sqrt[\beta+1]{\frac{N^{\beta} \mathcal{J}_{0}}{c_{\beta} g^{2} \tau_{c}^{2}}} .
$$

The resulting requirement on $N$, in order to keep the optimal narrow-band approximation and overlap only with the power-law tail

$$
\frac{t^{\mathrm{opt}}}{\pi N} \ll \tau_{c}, \frac{t^{\mathrm{opt}}}{\pi}
$$

is then

$$
N \gg \max \left\{\frac{\mathcal{J}_{0}}{c_{\beta} g^{2} \tau_{c}^{2} \pi^{\beta+1}}, 1\right\} .
$$

If the control is constrained (say, by maximum total energy $[26,27,91])$, it may happen that the filter function overlaps only with the Markovian region of the spectral density, $G_{\beta}^{M}\left(\tau_{c}, \omega\right) \approx \mathcal{A}_{\beta} \tau_{c}$, where it becomes a homogeneous function of the order of $\alpha=1$ on $\tau_{c}$. The attenuation factor is then independent of the dynamical control and is given by the Markovian limit

$$
\mathcal{J}^{M}\left(\tau_{c}, t\right)=g^{2} \mathcal{A}_{\beta} \tau_{c} t
$$

The ultimate bound (with $\alpha=1$ ) will then be achieved if the probe state is measured at $t_{\beta}^{M, \text { opt }}=$ $\left(\mathcal{J}_{0} / g^{2} \tau_{c}\right)$ independently of the control. This bound is always greater (worse) than the ultimate bound of Eq. (C3) for $\beta>2$. For $\beta=2$, they are equal.

(ii) For generalized Ohmic spectra $G_{s}$, the attenuation factor (D7) is

$$
\begin{aligned}
\mathcal{J}_{s}\left(\tau_{c}, t\right)= & g^{2} \sum_{k=1}^{k_{c}} F_{t}\left(\frac{\pi k N \tau_{c}}{t}\right) \\
& \times(s+1) \tau_{c}^{(s+1)}\left(\frac{\pi k N \tau_{c}}{t}\right)^{s} \\
= & \frac{c_{s} g^{2} \tau_{c}^{s+1} N^{s}}{t^{s-1}},
\end{aligned}
$$

where $c_{s}=\left\{\left[\pi^{s+1}(s+1)\right] / 2\right\} \sum_{k=1}^{k_{c}}(2 k-1)^{s-2}$ with $k_{c}=\left[t / \pi N \tau_{c}\right]$, where the square brackets denote the integer part. Since the harmonics that contribute with non-null terms are those below the cutoff, then $k_{c}$ is defined so as to satisfy $\left(\pi k_{c} N / t\right)<\omega_{c}<\left(\pi\left(k_{c}+\right.\right.$ 1) $N / t)$ and therefore $\left(t / \pi N \tau_{c}\right)+1<k_{c}<\left(t / \pi N \tau_{c}\right)$.

The first requirement to achieve the bound (C3) is to avoid any overlap between the filter and the cutoff region, i.e., $(t / \pi N k) \neq \tau_{c}$, with $k \in \mathbb{N}$. This is most likely to be satisfied under the narrow-band approximation that allows one to design the filter to be null at cutoff.

The second requirement is that the filter should overlap with the power-law region. Therefore, one needs at least one harmonic below the cutoff that avoids the overlap with the cutoff, i.e.,

$$
\frac{t}{\pi N}>\tau_{c} .
$$

Both conditions are ensured under $\mathrm{CW}$ control if $(t / \pi N)>\tau_{c}$, since the corresponding filter contains only one harmonic, leading to a simplified expression for the constant $c_{s}=\left[\pi^{s+1}(s+1)\right] / 2$. Then, $\mathcal{J}_{s}$ satisfies the equality in Eq. (C2), and the ultimate bound (C3) is achieved when $\mathcal{J}_{s}\left(\tau_{c}, t_{\text {opt }}\right)=\mathcal{J}_{0}$, yielding

$$
t_{s}^{\mathrm{opt}}=\tau_{c} \sqrt{s-1} \frac{c_{s} g^{2} \tau_{c}^{2} N^{s}}{\mathcal{J}_{0}} .
$$

To maintain this narrow-band approximation, with one harmonic below the cutoff frequency, i.e.,

$$
\frac{t_{s}^{\mathrm{opt}}}{\pi} \gg \frac{t_{s}^{\mathrm{opt}}}{\pi N}>\tau_{c}
$$

the number of cycles $N$ for super-Ohmic spectra $(s>1)$ should satisfy

$$
N_{\text {superOhm }}>\frac{\mathcal{J}_{0} \pi^{s-1}}{c_{s} g^{2} \tau_{c}^{2}} \quad \text { and } \quad N_{\text {superOhm }} \gg 1
$$

and for sub-Ohmic spectra $(0<s<1)$ 


$$
1 \ll N_{\text {subOhm }}<\frac{\mathcal{J}_{0} \pi^{s-1}}{c_{s} g^{2} \tau_{c}^{2}},
$$

which is attainable when $\sqrt{\left(\mathcal{J}_{0} \pi^{s-1} / c_{s}\right)} \gg g \tau_{c}$. For Ohmic spectra $(s=1)$

$$
1 \ll N_{\mathrm{Ohm}}=\frac{\mathcal{J}_{0}}{c_{s} g^{2} \tau_{c}^{2}},
$$

implying that it must satisfy $\sqrt{\frac{\mathcal{J}_{0}}{c_{s}}} \gg g \tau_{c}$.

When the last conditions cannot be satisfied, less restrictive optimal solutions can be found by demanding the filter function to be null at the cutoff frequency $\omega_{c}$ and then finding the optimal control time.

\section{c. The power-law exponent}

To estimate the power-law (PL) exponents $s$ and $\beta$ of spectral densities: $G^{\mathrm{PL}}(\omega) \propto \omega^{s}, \omega^{-\beta}$ or $G(\omega) \propto[1 /(1+$ $\left.a \omega^{\beta}\right)$ ] with $a=$ const, we define the exponent $x_{B}=\gamma$ that is common to both spectral densities: $\gamma=1+\beta$ or $\gamma=1-s$, we note that the derivative of the attenuation factor with respect to the exponent satisfies [cf. Eqs. (4) and (8) in the main text]

$$
\begin{aligned}
\left|\frac{\partial \mathcal{J}\left(x_{B}=\gamma, t\right)}{\partial \gamma}\right| & =\left|\int_{-\infty}^{\infty} d \omega F_{t}(\omega) \frac{\partial G(\gamma, \omega)}{\partial \gamma}\right| \\
& \leq\left|\int_{-\infty}^{\infty} d \omega F_{t}(\omega) \frac{\partial G^{\mathrm{PL}}(\gamma, \omega)}{\partial \gamma}\right| \\
& =\left|\frac{\partial \mathcal{J}^{\mathrm{PL}}(\gamma, t)}{\partial \gamma}\right| .
\end{aligned}
$$

This expression tightly bound the QFI (B4). To attain the ultimate bound for the relative error of the estimation by the maximized QFI, one needs to apply a control that probes only the power-law regime of the spectral density. The attenuation factor under such control can be expressed as $\mathcal{J}(\gamma, t)=\mathcal{J}^{\mathrm{PL}}(\gamma, t)=\left(t / T_{2}\right)^{\gamma}$, where the dephasing time $T_{2}$ depends on the applied control. Then,

$$
\left|\frac{\partial \mathcal{J}(\gamma, t)}{\partial \gamma}\right|=\frac{\mathcal{J}(\gamma, t)}{\gamma}|\ln (\mathcal{J}(\gamma, t))|
$$

and the ultimate precision bound in the estimation is then given by Eq. (13) of the main text. The bound is achieved when the qubit probe is measured at $t_{\mathrm{opt}}=T_{2} \sqrt[\gamma]{\mathcal{J}_{1}}$ with $\mathcal{J}_{1}=e^{-2.246} \approx 0.106$ when the dynamical control can ensure this regime. For example, under $\mathrm{CW}$ or $\mathrm{CPMG}$ control for estimating the power law of an OrnsteinUhlenbeck process, the time interval between the number of pulses should be smaller than the correlation time, $\left(t_{\text {opt }} / N\right) \ll \tau_{c}$, so that $N \gg\left(T_{2} \sqrt[v]{\mathcal{J}_{1}}\right) / \tau_{c}$.

\section{d. Unattainability of the ultimate bound under FID}

We here discuss the unattainability of the ultimate precision bound when the spin probe evolves freely, for the estimation of

(i) probe-bath interaction strength $g$ [the conditions to attain the relevant bound are discussed in the main text starting with Eqs. (D1) and ending in Eqs. (D3)] and

(ii) the correlation time $\tau_{c}$, for which, as observed in Fig. 2 of the main text, the estimation precision becomes worse as $g \tau_{c}$ grows. For the generalized Ornstein-Uhlenbeck spectra $G_{\beta}$, as discussed in the main text, the free-evolution filter $F_{t}^{\text {free }}(\omega)$, Eq. (D1), overlaps with the $\omega \approx 0$ region and consequently the equality in Eq. (C5) cannot be fulfilled. For the generalized Ohmic spectra $G_{s}$, when $t \ll \tau_{c}$, one can approximate Eq. (A12) by its zero-order term, considering that $F_{t}^{\text {free }}(\omega)$ is independent of $\omega$, since $G\left(\tau_{c}, \omega\right)$ is much narrower than $F_{t}^{\text {free }}(\omega)$.

The attenuation factor in this regime becomes independent of $\tau_{c}$, as in Eq. (D2): $\mathcal{J}_{\beta}^{\text {free }}\left(\tau_{c}, t\right)=\mathcal{J}_{s}^{\text {free }}\left(\tau_{c}, t\right) \approx \frac{1}{2} g^{2} t^{2}$. In this regime there is no information concerning the correlation time $\tau_{c}$. If $\frac{1}{2} g^{2} \tau_{c}^{2} \gg \mathcal{J}_{0}$, then it will not be possible to achieve the bound under free evolution. The attenuation factor will depend on $\tau_{c}$ only when $t \gtrsim \tau_{c}$, and therefore $\frac{1}{2} g^{2} t^{2} \gtrsim \frac{1}{2} g^{2} \tau_{c}^{2} \gg \mathcal{J}_{0}$, implying that the ultimate bound on Eq. (C3) cannot be achieved.

The limitation of the estimation of $\tau_{c}$ for the spectra $G_{s}$ using freely evolving spin probes is that the only control parameter is the time $t$ that may not simultaneously avoid the overlap of the filter function with $\omega_{c}$ and render the attenuation factor equal to $\mathcal{J}_{0}$, i.e., usually $t_{\text {opt }} \neq 4 \pi n \tau_{c}$, $n \in \mathbb{N}$. Hence, the ultimate bound is generally not achieved under free evolution.

\section{e. Pulse-error effects}

In the preceding analysis, we consider ideal, i.e., perfect and stroboscopic, pulses. If nonideal pulse effects are important, one needs a model for their effect on the dephasing. Typically, the pulses are applied with the same phase, as in CPMG, where flip-angle errors are compensated and the pure dephasing assumption is still suitable $[30,92]$. In this case, if finite-width pulse effects are significant, they can be considered by modifying the effective filter functions [93]. Therefore, within this model for the error effects, our ultimate bound is valid and achievable in the presence of such pulse-error effects. If pulse imperfections generate an effective Hamiltonian that deviates from a pure dephasing [92,94], then a different approach to calculate the ultimate bound must be pursued. However, for parameter estimation, control pulses with good fidelity are essential. Alternatively, one may have to resort to more elaborate controls, nicknamed by us selective dynamical recoupling, which we have shown to allow 
selective probing of the targeted noise source by factoring out uncontrollable noise sources, such as an intrinsic $T_{2}$ decoherence due to a white-noise (Markovian) process or due to pulse imperfections [10,11].

\section{APPENDIX E: INFORMATION GAIN FOR THE REAL-TIME ADAPTIVE-ESTIMATION PROTOCOL}

The best total control and measurement time $t^{\prime}$ to be chosen for the next iteration of the real-time estimation protocol are determined by the averaged information gain from the currently available probability distribution of $x_{B}$, $p\left(x_{B} \mid, d, t\right)$, assuming that experimental outcome $d$ is obtained in the iteration measured at $t$ [60].

The expected probability to obtain the outcome $d^{\prime}$ by measuring the controlled spin probe at time $t^{\prime}$ from the current probability distribution of $x_{B}$ is

$$
p\left(d^{\prime} \mid t^{\prime}, d, t\right)=\int p\left(d^{\prime} \mid x_{B}, t\right) p\left(x_{B} \mid, d, t\right) d \tau_{c} .
$$

The total information gain of a measurement at time $t^{\prime}$ is given by

$$
U\left(t^{\prime}\right)=\sum_{d^{\prime}} p\left(d^{\prime} \mid t^{\prime}, d, t\right) U\left(d^{\prime}, t^{\prime}\right),
$$

where $U\left(d^{\prime}, t^{\prime}\right)$ is the information gain if the measurement at $t^{\prime}$ gives the result $d^{\prime}$.

The information gain of an outcome, according to information theory, is measured by the entropy

$U\left(d^{\prime}, t^{\prime}\right)=\int p\left(x_{B} \mid d^{\prime}, t^{\prime}, d, t\right) \log \left[p\left(x_{B} \mid d^{\prime}, t^{\prime}, d, t\right)\right] d x_{B}$.

Then, the best time for the next control or measurement $t_{m}$ is defined by the value that maximizes the expected information

$$
\begin{aligned}
U\left(t_{m}\right)= & \max _{t^{\prime}}\left\{\sum_{d^{\prime}} p\left(d^{\prime} \mid t^{\prime}, d, t\right)\right. \\
& \left.\times \int p\left(x_{B} \mid d^{\prime}, t^{\prime}, d, t\right) \log \left[p\left(x_{B} \mid d^{\prime}, t^{\prime}, d, t\right)\right] d x_{B}\right\} .
\end{aligned}
$$

\section{APPENDIX F: EMULATION OF QND MEASUREMENTS INDUCED BY LASER-INDUCED DEPHASING IN NVC}

We propose to emulate QND measurements for NVC probes by laser-induced relaxation between the electronic ground and excited states.

As the probe state is initialized in the electronic ground state of the NVC, we probe the environment within this subspace. In order to mimic the QND measurements, one may use fast laser pulses for the transition from the ground to the excited electronic state, such that after $\sim 10 \mathrm{~ns}$ the electron spin is dephased in the excited electronic state, as shown in Ref. [77]. Then the radiative decay to the electronic ground states, which is (longitudinally) spin conserving, completes the emulation of a projective QND measurement in the $| \pm\rangle$ basis [78].

However, in a real experiment we may have to mimic imperfect, partial QND measurements. In particular, in real experiments we should consider that the radiative decay is circa 8 and 15 ns for the $| \pm 1\rangle$ and $|0\rangle$ electron spin states, respectively [95-97]. Concurrently, nonradiative decay of the $| \pm 1\rangle$ states to the ground electronic state will occur on a time scale of $300 \mathrm{~ns}$, mainly to the $|0\rangle$ spin state [78]. Therefore, the emulation of a QND measurement will not be perfect, and laser pulses have to be suitably optimized to enhance the estimation efficiency. Such specific optimization is beyond the scope of this work.

[1] J. M. Taylor, P. Cappellaro, L. Childress, L. Jiang, D. Budker, P. R. Hemmer, A. Yacoby, R. Walsworth, and M. D. Lukin, High-sensitivity diamond magnetometer with nanoscale resolution, Nat. Phys. 4, 810 (2008).

[2] Gopalakrishnan Balasubramanian, I. Y. Chan, Roman Kolesov, Mohannad Al-Hmoud, Julia Tisler, Chang Shin, Changdong Kim, Aleksander Wojcik, Philip R. Hemmer, Anke Krueger, Tobias Hanke, Alfred Leitenstorfer, Rudolf Bratschitsch, Fedor Jelezko, and Jörg Wrachtrup, Nanoscale imaging magnetometry with diamond spins under ambient conditions, Nature (London) 455, 648 (2008).

[3] G. Kucsko, P. C. Maurer, N. Y. Yao, M. Kubo, H. J. Noh, P. K. Lo, H. Park, and M. D. Lukin, Nanometre-scale thermometry in a living cell, Nature (London) 500, 54 (2013).

[4] P. Neumann, I. Jakobi, F. Dolde, C. Burk, R. Reuter, G. Waldherr, J. Honert, T. Wolf, A. Brunner, J. H. Shim, D. Suter, H. Sumiya, J. Isoya, and J. Wrachtrup, High-precision nanoscale temperature sensing using single defects in diamond, Nano Lett. 13, 2738 (2013).

[5] David M. Toyli, Charles F. de las Casas, David J. Christle, Viatcheslav V. Dobrovitski, and David D. Awschalom, Fluorescence thermometry enhanced by the quantum coherence of single spins in diamond, Proc. Natl. Acad. Sci. U.S.A. 110, 8417 (2013).

[6] S. Steinert, F. Ziem, L. T. Hall, A. Zappe, M. Schweikert, N. Götz, A. Aird, G. Balasubramanian, L. Hollenberg, and J. Wrachtrup, Magnetic spin imaging under ambient conditions with sub-cellular resolution, Nat. Commun. 4, 1607 (2013).

[7] M. S. Grinolds, M. Warner, K. De Greve, Y. Dovzhenko, L. Thiel, R. L. Walsworth, S. Hong, P. Maletinsky, and A. Yacoby, Subnanometre resolution in three-dimensional magnetic resonance imaging of individual dark spins, Nat. Nanotechnol. 9, 279 (2014).

[8] A. O. Sushkov, I. Lovchinsky, N. Chisholm, R. L. Walsworth, H. Park, and M. D. Lukin, Magnetic Resonance Detection of Individual Proton Spins Using Quantum Reporters, Phys. Rev. Lett. 113, 197601 (2014). 
[9] A. Mittermaier and L. E. Kay, New tools provide new insights in NMR studies of protein dynamics, Science 312, 224 (2006).

[10] Pieter E.S. Smith, Guy Bensky, Gonzalo A. Álvarez, Gershon Kurizki, and Lucio Frydman, Shift-driven modulations of spin-echo signals, Proc. Natl. Acad. Sci. U.S.A. 109, 5958 (2012).

[11] Gonzalo A. Álvarez, Noam Shemesh, and Lucio Frydman, Coherent Dynamical Recoupling of Diffusion-Driven Decoherence in Magnetic Resonance, Phys. Rev. Lett. 111, 080404 (2013).

[12] Wojciech Zurek, Decoherence, einselection, and the quantum origins of the classical, Rev. Mod. Phys. 75, 715 (2003).

[13] L. Viola, E. Knill, and S. Lloyd, Dynamical Decoupling of Open Quantum Systems, Phys. Rev. Lett. 82, 2417 (1999).

[14] L. Viola, S. Lloyd, and E. Knill, Universal Control of Decoupled Quantum Systems, Phys. Rev. Lett. 83, 4888 (1999).

[15] A. G. Kofman and G. Kurizki, Universal Dynamical Control of Quantum Mechanical Decay: Modulation of the Coupling to the Continuum, Phys. Rev. Lett. 87, 270405 (2001).

[16] A. G. Kofman and G. Kurizki, Unified Theory of Dynamically Suppressed Qubit Decoherence in Thermal Baths, Phys. Rev. Lett. 93, 130406 (2004).

[17] Goren Gordon, Noam Erez, and Gershon Kurizki, Universal dynamical decoherence control of noisy single- and multiqubit systems, J. Phys. B 40, S75 (2007).

[18] K. Khodjasteh and D. A. Lidar, Fault-Tolerant Quantum Dynamical Decoupling, Phys. Rev. Lett. 95, 180501 (2005).

[19] Alexandre M. Souza, Gonzalo A. Álvarez, and Dieter Suter, Robust dynamical decoupling, Phil. Trans. R. Soc. A 370, 4748 (2012).

[20] Carlos A. Meriles, Liang Jiang, Garry Goldstein, Jonathan S. Hodges, Jeronimo Maze, Mikhail D. Lukin, and Paola Cappellaro, Imaging mesoscopic nuclear spin noise with a diamond magnetometer, J. Chem. Phys. 133, 124105 (2010).

[21] Ido Almog, Yoav Sagi, Goren Gordon, Guy Bensky, Gershon Kurizki, and Nir Davidson, Direct measurement of the system-environment coupling as a tool for understanding decoherence and dynamical decoupling, J. Phys. B 44, 154006 (2011).

[22] Jonas Bylander, Simon Gustavsson, Fei Yan, Fumiki Yoshihara, Khalil Harrabi, George Fitch, David G. Cory, Yasunobu Nakamura, Jaw-Shen Tsai, and William D. Oliver, Noise spectroscopy through dynamical decoupling with a superconducting flux qubit, Nat. Phys. 7, 565 (2011).

[23] Gonzalo A. Álvarez and Dieter Suter, Measuring the Spectrum of Colored Noise by Dynamical Decoupling, Phys. Rev. Lett. 107, 230501 (2011).

[24] Christian O. Bretschneider, Gonzalo A. Álvarez, Gershon Kurizki, and Lucio Frydman, Controlling Spin-Spin Network Dynamics by Repeated Projective Measurements, Phys. Rev. Lett. 108, 140403 (2012).

[25] Lukasz Cywinski, Dynamical-decoupling noise spectroscopy at an optimal working point of a qubit, Phys. Rev. A 90, 042307 (2014).

[26] Jens Clausen, Guy Bensky, and Gershon Kurizki, Bath-Optimized Minimal-Energy Protection of Quantum
Operations from Decoherence, Phys. Rev. Lett. 104, 040401 (2010).

[27] Goren Gordon, Gershon Kurizki, and Daniel A. Lidar, Optimal Dynamical Decoherence Control of a Qubit, Phys. Rev. Lett. 101, 010403 (2008).

[28] Hermann Uys, Michael J. Biercuk, and John J. Bollinger, Optimized Noise Filtration through Dynamical Decoupling, Phys. Rev. Lett. 103, 040501 (2009).

[29] K. Khodjasteh, D. A. Lidar, and L. Viola, Arbitrarily Accurate Dynamical Control in Open Quantum Systems, Phys. Rev. Lett. 104, 090501 (2010).

[30] Ashok Ajoy, Gonzalo A. Álvarez, and Dieter Suter, Optimal pulse spacing for dynamical decoupling in the presence of a purely dephasing spin bath, Phys. Rev. A 83, 032303 (2011).

[31] David Hayes, Kaveh Khodjasteh, Lorenza Viola, and Michael J. Biercuk, Reducing sequencing complexity in dynamical quantum error suppression by Walsh modulation, Phys. Rev. A 84, 062323 (2011).

[32] Victor Mukherjee, Alberto Carlini, Andrea Mari, Tommaso Caneva, Simone Montangero, Tommaso Calarco, Rosario Fazio, and Vittorio Giovannetti, Speeding up and slowing down the relaxation of a qubit by optimal control, Phys. Rev. A 88, 062326 (2013).

[33] Kaveh Khodjasteh, Jarrah Sastrawan, David Hayes, Todd J. Green, Michael J. Biercuk, and Lorenza Viola, Designing a practical high-fidelity long-time quantum memory, Nat. Commun. 4, 2045 (2013).

[34] G. Gordon and G. Kurizki, Dynamical protection of quantum computation from decoherence in laser-driven cold-ion and cold-atom systems, New J. Phys. 10, 045005 (2008).

[35] K. Khodjasteh and L. Viola, Dynamically Error-Corrected Gates for Universal Quantum Computation, Phys. Rev. Lett. 102, 080501 (2009).

[36] Gerardo A. Paz-Silva, A. T. Rezakhani, Jason M. Dominy, and D. A. Lidar, Zeno Effect for Quantum Computation and Control, Phys. Rev. Lett. 108, 080501 (2012).

[37] Chingiz Kabytayev, Todd J. Green, Kaveh Khodjasteh, Michael J. Biercuk, Lorenza Viola, and Kenneth R. Brown, Robustness of composite pulses to time-dependent control noise, Phys. Rev. A 90, 012316 (2014).

[38] Gonzalo A. Álvarez, Mor Mishkovsky, Ernesto P. Danieli, Patricia R. Levstein, Horacio M. Pastawski, and Lucio Frydman, Perfect state transfers by selective quantum interferences within complex spin networks, Phys. Rev. A 81, 060302(R) (2010).

[39] Analia Zwick, Gonzalo A Álvarez, Guy Bensky, and Gershon Kurizki, Optimized dynamical control of state transfer through noisy spin chains, New J. Phys. 16, 065021 (2014).

[40] David Petrosyan, Guy Bensky, Gershon Kurizki, Igor Mazets, Johannes Majer, and Jörg Schmiedmayer, Reversible state transfer between superconducting qubits and atomic ensembles, Phys. Rev. A 79, 040304 (2009).

[41] Guy Bensky, David Petrosyan, Johannes Majer, Jörg Schmiedmayer, and Gershon Kurizki, Optimizing inhomogeneous spin ensembles for quantum memory, Phys. Rev. A 86, 012310 (2012).

[42] Noam Shemesh, Gonzalo A. Álvarez, and Lucio Frydman, Measuring small compartment dimensions by probing 
diffusion dynamics via non-uniform oscillating-gradient spin-echo (NOGSE) NMR, J. Magn. Reson. 237, 49 (2013).

[43] H. J. Mamin, M. Kim, M. H. Sherwood, C. T. Rettner, K. Ohno, D. D. Awschalom, and D. Rugar, Nanoscale nuclear magnetic resonance with a nitrogen-vacancy spin sensor, Science 339, 557 (2013).

[44] T. Staudacher, F. Shi, S. Pezzagna, J. Meijer, J. Du, C. A. Meriles, F. Reinhard, and J. Wrachtrup, Nuclear magnetic resonance spectroscopy on a (5-nanometer $)^{3}$ sample volume, Science 339, 561 (2013).

[45] B. M. Escher, R. L. de Matos Filho, and L. Davidovich, General framework for estimating the ultimate precision limit in noisy quantum-enhanced metrology, Nat. Phys. 7, 406 (2011).

[46] Samuel L. Braunstein and Carlton M. Caves, Statistical Distance and the Geometry of Quantum States, Phys. Rev. Lett. 72, 3439 (1994).

[47] Matteo G. A. Paris, Quantum estimation for quantum technology, Int. J. Quantum. Inform. 07, 125 (2009).

[48] Claudia Benedetti and Matteo G. A. Paris, Characterization of classical gaussian processes using quantum probes, Phys. Lett. A 378, 2495 (2014).

[49] Claudia Benedetti, Fabrizio Buscemi, Paolo Bordone, and Matteo G. A. Paris, Quantum probes for the spectral properties of a classical environment, Phys. Rev. A 89, 032114 (2014).

[50] Mihai D. Vidrighin, Gaia Donati, Marco G. Genoni, Xian-Min Jin, W. Steven Kolthammer, M. S. Kim, Animesh Datta, Marco Barbieri, and Ian A. Walmsley, Joint estimation of phase and phase diffusion for quantum metrology, Nat. Commun. 5, 3532 (2014).

[51] Luis A. Correa, Mohammad Mehboudi, Gerardo Adesso, and Anna Sanpera, Individual Quantum Probes for Optimal Thermometry, Phys. Rev. Lett. 114, 220405 (2015).

[52] A. G. Kofman and G. Kurizki, Acceleration of quantum decay processes by frequent observations, Nature (London) 405, 546 (2000).

[53] S. F. Huelga, C. Macchiavello, T. Pellizzari, A. K. Ekert, M. B. Plenio, and J. I. Cirac, Improvement of Frequency Standards with Quantum Entanglement, Phys. Rev. Lett. 79, 3865 (1997).

[54] N. Bar-Gill, L. M. Pham, C. Belthangady, D. Le Sage, P. Cappellaro, J. R. Maze, M. D. Lukin, A. Yacoby, and R. Walsworth, Suppression of spin-bath dynamics for improved coherence of multi-spin-qubit systems, Nat. Commun. 3, 858 (2012).

[55] J. Medford, Ł. Cywiński, C. Barthel, C. M. Marcus, M. P. Hanson, and A. C. Gossard, Scaling of Dynamical Decoupling for Spin Qubits, Phys. Rev. Lett. 108, 086802 (2012).

[56] Y. Romach, C. Müller, T. Unden, L. J. Rogers, T. Isoda, K. M. Itoh, M. Markham, A. Stacey, J. Meijer, S. Pezzagna, B. Naydenov, L. P. McGuinness, N. Bar-Gill, and F. Jelezko, Spectroscopy of Surface-Induced Noise Using Shallow Spins in Diamond, Phys. Rev. Lett. 114, 017601 (2015).

[57] G. De Lange, Z. H. Wang, D. Riste, V. V. Dobrovitski, and R. Hanson, Universal dynamical decoupling of a single solid-state spin from a spin bath, Science 330, 60 (2010).

[58] D. Suter and R. R. Ernst, Spin diffusion in resolved solidstate NMR spectra, Phys. Rev. B 32, 5608 (1985).
[59] A. Feintuch, A. Grayevsky, N. Kaplan, and E. Dormann, Diffusive Diffraction of the Local ESR Pulse-Gradient SpinEcho Signal in a Restricted One-Dimensional Conductor, Phys. Rev. Lett. 92, 156803 (2004).

[60] Christopher E. Granade, Christopher Ferrie, Nathan Wiebe, and D. G. Cory, Robust online Hamiltonian learning, New J. Phys. 14, 103013 (2012).

[61] Alexandr Sergeevich, Anushya Chandran, Joshua Combes, Stephen D. Bartlett, and Howard M. Wiseman, Characterization of a qubit Hamiltonian using adaptive measurements in a fixed basis, Phys. Rev. A 84, 052315 (2011).

[62] M. D. Shulman, S. P. Harvey, J. M. Nichol, S. D. Bartlett, A. C. Doherty, V. Umansky, and A. Yacoby, Suppressing qubit dephasing using real-time Hamiltonian estimation, Nat. Commun. 5, 5156 (2014).

[63] Paola Cappellaro, Spin-bath narrowing with adaptive parameter estimation, Phys. Rev. A 85, 030301 (2012).

[64] Gonzalo A. Álvarez, Noam Shemesh, and Lucio Frydman, Diffusion-assisted selective dynamical recoupling: A new approach to measure background gradients in magnetic resonance, J. Chem. Phys. 140, 084205 (2014).

[65] A. Meda, A. Caprile, A. Avella, I. Ruo Berchera, I. P. Degiovanni, A. Magni, and M. Genovese, Magneto-optical imaging technique for hostile environments: The ghost imaging approach, Appl. Phys. Lett. 106, 262405 (2015).

[66] Noam Shemesh, Gonzalo A. Álvarez, and Lucio Frydman, Size distribution imaging by non-uniform oscillatinggradient spin echo (NOGSE) MRI, PLoS One 10, e0133201 (2015).

[67] Heinz-Peter Breuer and Francesco Petruccione, The Theory of Open Quantum Systems (Oxford University, New York, 2007).

[68] Gonzalo A. Álvarez, D. D. Bhaktavatsala Rao, Lucio Frydman, and Gershon Kurizki, Zeno and Anti-Zeno Polarization Control of Spin Ensembles by Induced Dephasing, Phys. Rev. Lett. 105, 160401 (2010).

[69] Charles P. Slichter, Principles of Magnetic Resonance (Springer, Berlin, 1990).

[70] D. Meshulach, D. Yelin, and Y. Silberberg, Adaptive realtime femtosecond pulse shaping, J. Opt. Soc. Am. B 15, 1615 (1998).

[71] Adriano A. Berni, Tobias Gehring, Bo M. Nielsen, Vitus Händchen, Matteo G. A. Paris, and Ulrik L. Andersen, $A b$ initio quantum-enhanced optical phase estimation using real-time feedback control, Nat. Photonics 9, 577 (2015).

[72] R. Vijay, C. Macklin, D. H. Slichter, S. J. Weber, K. W. Murch, R. Naik, A. N. Korotkov, and I. Siddiqi, Stabilizing Rabi oscillations in a superconducting qubit using quantum feedback, Nature (London) 490, 77 (2012).

[73] Goren Gordon, Noam Erez, and Gershon Kurizki, Universal dynamical decoherence control of noisy single- and multiqubit systems, J. Phys. B 40, S75 (2007).

[74] Robert Tycko, Stochastic Dipolar Recoupling in Nuclear Magnetic Resonance of Solids, Phys. Rev. Lett. 99, 187601 (2007).

[75] Jean Dalibard, Yvan Castin, and Klaus Mølmer, WaveFunction Approach to Dissipative Processes in Quantum Optics, Phys. Rev. Lett. 68, 580 (1992).

[76] G. Teklemariam, E. M. Fortunato, C. C. Lopez, J. Emerson, Juan Pablo Paz, T. F. Havel, and D. G. Cory, Method for 
modeling decoherence on a quantum-information processor, Phys. Rev. A 67, 062316 (2003).

[77] G. D. Fuchs, V. V. Dobrovitski, D. M. Toyli, F. J. Heremans, C. D. Weis, T. Schenkel, and D. D. Awschalom, Excitedstate spin coherence of a single nitrogen-vacancy centre in diamond, Nat. Phys. 6, 668 (2010).

[78] N. B. Manson, J. P. Harrison, and M. J. Sellars, Nitrogenvacancy center in diamond: Model of the electronic structure and associated dynamics, Phys. Rev. B 74, 104303 (2006).

[79] Gonzalo A. Álvarez, Robin Kaiser, and Dieter Suter, Quantum simulations of localization effects with dipolar interactions, Ann. Phys. (Berlin) 525, 833 (2013).

[80] Gonzalo A. Álvarez, Dieter Suter, and Robin Kaiser, Localization-delocalization transition in the dynamics of dipolar-coupled nuclear spins, Science 349, 846 (2015).

[81] C. Joachim, J. K. Gimzewski, and A. Aviram, Electronics using hybrid-molecular and mono-molecular devices, Nature (London) 408, 541 (2000).

[82] G. Ithier, E. Collin, P. Joyez, P. J. Meeson, D. Vion, D. Esteve, F. Chiarello, A. Shnirman, Y. Makhlin, J. Schriefl, and G. Schön, Decoherence in a superconducting quantum bit circuit, Phys. Rev. B 72, 134519 (2005).

[83] Vittorio Giovannetti, Seth Lloyd, and Lorenzo Maccone, Quantum Metrology, Phys. Rev. Lett. 96, 010401 (2006).

[84] Matteo A.C. Rossi and Matteo G. A. Paris, Entangled quantum probes for dynamical environmental noise, Phys. Rev. A 92, 010302 (2015).

[85] G. Gordon and G. Kurizki, Preventing Multipartite Disentanglement by Local Modulations, Phys. Rev. Lett. 97, 110503 (2006).

[86] Goren Gordon and Gershon Kurizki, Scalability of decoherence control in entangled systems, Phys. Rev. A 83, 032321 (2011).

[87] Mankei Tsang, Howard M. Wiseman, and Carlton M. Caves, Fundamental Quantum Limit to Waveform Estimation, Phys. Rev. Lett. 106, 090401 (2011).
[88] A. G. Kofman and G. Kurizki, Theory of dynamical control of qubit decay and decoherence, IEEE Trans. Nanotechnol. 4, 116 (2005).

[89] Goren Gordon, Dynamical decoherence control of multipartite systems, J. Phys. B 42, 223001 (2009).

[90] D. D. Bhaktavatsala Rao and Gershon Kurizki, From Zeno to anti-Zeno regime: Decoherence-control dependence on the quantum statistics of the bath, Phys. Rev. A 83, 032105 (2011).

[91] Jens Clausen, Guy Bensky, and Gershon Kurizki, Taskoptimized control of open quantum systems, Phys. Rev. A 85, 052105 (2012).

[92] Gonzalo A. Álvarez, Ashok Ajoy, Xinhua Peng, and Dieter Suter, Performance comparison of dynamical decoupling sequences for a qubit in a rapidly fluctuating spin bath, Phys. Rev. A 82, 042306 (2010).

[93] Michael J. Biercuk, Hermann Uys, Aaron P. VanDevender, Nobuyasu Shiga, Wayne M. Itano, and John J. Bollinger, Experimental Uhrig dynamical decoupling using trapped ions, Phys. Rev. A 79, 062324 (2009).

[94] K. Khodjasteh and D. A. Lidar, Performance of deterministic dynamical decoupling schemes: Concatenated and periodic pulse sequences, Phys. Rev. A 75, 062310 (2007).

[95] A. Batalov, C. Zierl, T. Gaebel, P. Neumann, I.-Y. Chan, G. Balasubramanian, P. R. Hemmer, F. Jelezko, and J. Wrachtrup, Temporal Coherence of Photons Emitted by Single Nitrogen-Vacancy Defect Centers in Diamond Using Optical Rabi-Oscillations, Phys. Rev. Lett. 100, 077401 (2008).

[96] Lucio Robledo, Hannes Bernien, Toeno van der Sar, and Ronald Hanson, Spin dynamics in the optical cycle of single nitrogen-vacancy centres in diamond, New J. Phys. 13, 025013 (2011).

[97] G. D. Fuchs, A. L. Falk, V. V. Dobrovitski, and D. D. Awschalom, Spin Coherence during Optical Excitation of a Single Nitrogen-Vacancy Center in Diamond, Phys. Rev. Lett. 108, 157602 (2012). 